\title{
THE ERGODIC THEORY OF DISCRETE ISOMETRY GROUPS ON MANIFOLDS OF VARIABLE NEGATIVE CURVATURE
}

\author{
CHENGBO YUE
}

\begin{abstract}
This paper studies the ergodic theory at infinity of an arbitrary discrete isometry group $\Gamma$ acting on any Hadamard manifold $H$ of pinched variable negative curvature. Most of the results obtained by Sullivan in the constant curvature case are generalized to the case of variable curvature. We describe connections between measures supported on the limit set of $\Gamma$, dynamics of the geodesic flow and the geometry of $M=H / \Gamma$. We explore the relationship between the growth exponent of the group, the Hausdorff dimension of the limit set and the topological entropy of the geodesic flow. The equivalence of various descriptions of an analogue of the Hopf dichotomy is proved. As applications, we settle a question of J. Feldman and M. Ratner about the horocycle flow on a finite volume surface of negative curvature and obtain an asymptotic formula for the counting function of lattice points. At the end of this paper, we apply our results to the study of some rigidity problems. More applications to Mostow rigidity for discrete subgroups of rank 1 noncompact semisimple Lie groups with infinite covolume will be published in subsequent papers by the author.
\end{abstract}

\section{NOTATIONS}

- $\quad H$ is a Hadamard manifold with pinched sectional curvature

$$
-K_{2}^{2} \leq K \leq-K_{1}^{2}, \quad K_{2} \geq K_{1}>0 .
$$

- $\quad \Gamma$ is a (non-elementary) discrete isometry group acting on $H$ freely and properly discontinuously (which is called Fuchsian).

- $\partial H$ is the ideal boundary of $H$.

- $\quad M=H / \Gamma$ is a complete Riemannian manifold.

- $S H$ (or $S M$ ) is the unit tangent bundle.

- $g^{t}$ is the geodesic flow on $S H$ (or $S M$ ).

- $v(t)$ is the unique geodesic in $S H$ (resp. $S M$ ) with the initial velocity $\dot{v}(0)=v \in S H($ resp. $S M)$.

- $v(\infty)$ (resp. $v(-\infty)$ ) is the asymptotic class of the geodesic $v(t)$ (resp. $v(-t))$.

- $\quad L(\Gamma)$ is the limit set of $\Gamma$ on $\partial H$.

- $\quad L^{r}(\Gamma)$ is the radical limit set of $\Gamma$ in $L(\Gamma)$.

- $\Omega=\Omega(\Gamma)$ is the nonwandering set of the geodesic flow on $S M$

Received by the editors May 7, 1995 .

1991 Mathematics Subject Classification. Primary 58F17; Secondary 58F11, 58F15, 20H10.

Research at MSRI supported by NSF Grant \#DMS 8505550 . Also partially supported by NSF Grant \#DMS 9403870 and SFB 170. 


\section{INTRODUCTION}

The purpose of this paper is twofold. We first want to built up a detailed ergodic theory of discrete isometry subgroups of negatively curved Riemannian manifolds. Our main motivation, however, is to lay a necessary foundation for our generalizations of Mostow rigidity ([Yu3][Yu4]).

Assume that $H$ is a simply connected $n$-dimensional Riemannian manifold of pinched negative curvature $-\infty<-K_{2}^{2} \leq K \leq-K_{1}^{2}<0$. We know that $H$ is diffeomorphic to an open disc $D^{n}$ and the boundary of $H$ at infinity is homeomorphic to a sphere $S^{n-1}$. Throughout this paper we always assume that $\Gamma$ is a torsion free non-elementary discrete isometry group acting on $H$ freely and properly discontinuously. For the sake of simplicity, we call a group satisfying these properties Fuchsian. This terminology was due to Eberlein and O'Neil in their pioneering work [E-O'N]. Imagine that we stand inside the disc, looking at an orbit $\Gamma x$ of $\Gamma$ and thinking of each orbit point as a star; then we see a galaxy on the sky at infinity, which is called the limit set of the Fuchsian group and is denoted by $L(\Gamma)$. We want to read information about the geometry of the quotient manifold $M=H / \Gamma$ by looking at this galaxy. The group acts naturally on its limit set. We also want to study this action by looking at the dynamics of the geodesic flow $g^{t}$ on $M$ and vice versa. Actually, all these problems have their origin in the theory of classical Fuchsian and Kleinian groups. Inspired by the classical theory, Patrick Eberlein published a series of papers since the early seventies. He had achieved, more or less, a complete picture of the topological dynamics of the $\Gamma$ action and the geodesic flow. However, he did not study the ergodic theory of the $\Gamma$ action and the geodesic flow. Indeed, the corresponding ergodic theory turns out to be much more complicated, and much more interesting. The main theme of this paper is to build a foundation of that theory.

Apart from Eberlein's work, there are two other sources which influenced the present paper. Our most important inspiration came from a group of Sullivan's papers (see [S1], [S2] and the references there). For me his works in the constant curvature case are almost the axioms for a corresponding theory in the variable curvature case. The generalizations are sometimes easy and straightforward, sometimes much more complicated and difficult, but we never lose track or direction. The other influence came from Gromov's fundamental paper [G] and a series of researches after it, especially [C]. In several occasions results for the more abstract Gromov hyperbolic space helped shorten our route, but theories for concrete objects are always richer, more precise and more beautiful.

(0.1) Densities on the limit set. To measure the thickness of the galaxy, we introduce two classes of measures on $L(\Gamma)$.

(0.1.A) The conformal density. The conformal density is constructed by Patterson in the case where $\operatorname{dim} H=2, K \equiv-1$ and his construction was generalized by Sullivan to the case where $K \equiv-1$ in all dimensions. One can easily perform the same construction in the variable curvature case. Namely, consider the Poincaré series

$$
g_{s}(x, y)=\sum_{\gamma \in \Gamma} e^{-s d(x, \gamma y)}
$$


and define the critical exponent $\delta(\Gamma) \triangleq \delta$ to be the supremum of all $s$ such that the series diverges at $s$. Then by averaging the Dirac measure at each lattice point we get the conformal density $\sigma_{y}$ as a weak limit

$$
\sigma_{y}=\lim _{s_{i} \rightarrow \delta+} \frac{\sum_{\gamma \in \Gamma} e^{-s_{i} d(x, \gamma y)} \operatorname{Dirac}(\gamma y)}{\sum_{\gamma \in \Gamma} e^{-s_{i} d(x, \gamma y)}},
$$

which is supported on $L(\Gamma)$ (after a minor adjustment, see $[\mathrm{P}]$ ) and satisfies

$$
\frac{d \sigma_{y}}{d \sigma_{x}}(\zeta)=e^{-\delta \rho_{x, \zeta}(y)}
$$

where $\rho_{x, \zeta}$ is the Busemann function at $\zeta$ with $\rho_{x, \zeta}(x)=0$.

(0.1.B) The harmonic density (after Ancona [A2]). Let $\lambda_{1}$ be the bottom of the spectrum of the Laplacian on $L^{2}(H)$. Then for each $0 \leq \lambda<\lambda_{1}$ there is a nice $\lambda$-potential theory (see $[\mathrm{A} 1])$. Let $G_{\lambda}(x, y)$ and $K_{\lambda}(x, y, \zeta)$ be the Green kernel and the Poisson kernel of the operator $\triangle+\lambda I$ and consider the Green series

$$
\sum_{\gamma \in \Gamma} G_{\lambda}\left(x_{0}, \gamma y\right)
$$

Define the critical eigenvalue $\lambda(\Gamma)$ to be the supremum of all $\lambda$ such that the Green series converges. Then we get the harmonic density $\tau_{y}$ as a weak limit of the following average:

$$
\lim _{\lambda_{i} \rightarrow \lambda(\Gamma)^{-}} \frac{\sum_{\gamma \in \Gamma} G_{\lambda_{i}}\left(x_{0}, \gamma y\right) \operatorname{Dirac}(\gamma y)}{\sum_{\gamma \in \Gamma} G_{\lambda_{i}}\left(x_{0}, \gamma x\right)},
$$

which is supported on $L(\Gamma)$ (after a minor adjustment, see [A2]) and satisfies

$$
\frac{d \tau_{y}}{d \tau_{x}}(\zeta)=K_{\lambda(\Gamma)}(x, y, \zeta)
$$

(0.2) The ergodic theory. There are close connections between the geodesic flow $g^{t}$ on $S M$ and the limit set $\Gamma$. For example

a) The nonwandering set $\Omega$ (see (5.1) for definition) of $g^{t}$ on $S M$ corresponds to $(L(\Gamma) \times L(\Gamma) \backslash$ diagonal $) / \Gamma$; i.e., $v \in \Omega$ iff there is a lift $\tilde{v} \in S H$ of $v$, such that $\tilde{v}(\infty) \in L(\Gamma)$ and $\tilde{v}(-\infty) \in L(\Gamma)$.

b) A point $v \in S M$ is $g^{t}$-conservative (i.e., $g^{t} v$ returns to a compact set infinitely often as $t \rightarrow+\infty)$ iff $v$ lifts to $\tilde{v} \in S H$ such that $\tilde{v}(\infty)$ belongs to the radial limit set $L^{r}(\Gamma) \subset L(\Gamma)$ (see (3.5) for definitions).

c) There is a one-one correspondence between the $g^{t}$-invariant (resp. ergodic) measures on $\Omega$ and the $\Gamma$-invariant (resp. ergodic) measures on $L(\Gamma) \times L(\Gamma)$.

(0.2.A) From the conformal density $\sigma$ we construct a $g^{t}$-invariant measure $\mu^{\sigma}$ on $\Omega$ (after Sullivan, Kaimanovich). We then prove the equivalence of the following dichotomies:

- The geodesic flow is either completely conservative with respect to $\mu^{\sigma}$ or completely dissipative.

- $\Gamma$ is either of divergent type (i.e., the Poincaré series diverges at $\delta(\Gamma)$ ) or of convergent type (i.e., the Poincaré series converges at $\delta(\Gamma)$ ).

Theorem A. The following properties are equivalent: 
(1) $\Gamma$ is of divergent type.

(2) $\sigma\left(L^{r}(\Gamma)\right)>0$.

(3) $L^{r}(\Gamma)$ has full $\sigma$-measure.

(4) The geodesic flow is ergodic with respect to $\mu^{\sigma}$.

(0.2.B) From the harmonic density $\tau$ we construct a $g^{t}$-invariant measure $\nu^{\tau}$ on $\Omega$. We also have the equivalence of the following dichotomies (assuming $\lambda(\Gamma)<\lambda_{1}$ ):

- The geodesic flow is either completely conservative with respect to the measure $\nu^{\tau}$ or completely dissipative.

-• The Green series is either divergent at $\lambda(\Gamma)$ or convergent at $\lambda(\Gamma)$.

Theorem B. Assuming $\lambda(\Gamma)<\lambda_{1}$, then the following properties are equivalent:

(1) The Green series diverges at $\lambda(\Gamma)$.

(2) $\tau\left(L^{r}(\Gamma)\right)>0$.

(3) $L^{r}(\Gamma)$ has full $\tau$-measure.

(4) The geodesic flow $g^{t}$ is ergodic with respect to $\nu^{\tau}$.

The proof of Theorem A is achieved by the Sullivan shadow lemma in the variable curvature case. The proof of Theorem B uses a biased Brownian motion argument.

(0.3) Hausdorff dimensions of the limit set. We introduce four metrics on $\partial H$ (after Gromov, Kaimanovich) which are mutually equivalent. Some of our arguments prefer one metric and some prefer another. We then study the Hausdorff dimension $H D$ of the limit set with respect to these equivalent metrics. One of our main theorems is the following

Theorem C. Assuming that $\Gamma$ is a general Fuchsian group, then we have:

(1) The Hausdorff dimension of the radial limit set is always $\leq \delta(\Gamma)$.

(2) If $\mu^{\sigma}(S M)<\infty$, then $H D\left(L^{r}(\Gamma)\right)=\delta(\Gamma)$.

Remarks. If $\mu^{\sigma}(S M)<\infty$ (resp. $\left.\nu^{\tau}(S M)<\infty\right)$, then by the Poincaré recurrence theorem, the geodesic flow must be completely conservative and $\Gamma$ must be of divergent type. We also prove the following finiteness criterion which is used later in the lattice case.

Theorem D. If the Poincaré series satisfies

$$
\sum_{\gamma \in \Gamma} e^{-s d(x, \gamma x)} \geq \frac{A}{s-\delta},
$$

for $s>\delta=\delta(\Gamma)$ and for some constant $A>0$, then $\mu^{\sigma}(S M)<\infty$.

(0.4) Convex cocompact groups. A Fuchsian group $\Gamma$ is convex cocompact if and only if its corresponding nonwandering set $\Omega$ is compact. In this case, all the thermodynamic formalisms apply. In particular, for each Hölder continuous function $\varphi: \Omega \rightarrow R$ there corresponds a unique probability measure $\mu_{\varphi}$ on $\Omega$ (which is called the equilibrium state of $\varphi$ ), such that $\left(g^{t}, \mu_{\varphi}\right)$ is Bernoulli. We identify $\mu^{\sigma}$ and $\nu^{\tau}$ as equilibrium states of some special functions. For each equilibrium state $\mu_{\varphi}$ there corresponds a unique measure class $\partial \mu_{\varphi}$ on $\partial H$. We have the following

Theorem E. If $\Gamma$ is convex cocompact, then we have

(1) $H D(L(\Gamma))=\delta$.

(2) The conformal density $\sigma_{x}$ is precisely the $\delta$-dimensional Hausdorff measure of the Busemann metric. 
(3) The lattice counting function $N(x, y, R)=\#\{\Gamma x \cap B(y, R)\}$ satisfies $N(x, y, R)$ $\sim A c(x) c(y) e^{\delta R}$, where $A$ is a constant not depending on $x, y$, and $c(x)=$ $\sigma_{x}(\partial H)$.

(4) The Hausdorff dimension of the measure class $\partial \mu_{\varphi}$ is exactly the metric entropy of the geodesic flow with respect to the invariant measure $\mu_{\varphi}$.

(0.5) Geometrically finite groups and lattices. In the variable curvature case, geometrically finite groups are still well-defined for discrete groups. Unlike the constant curvature case, we do not have a good ergodic theory for this class of groups. Even for the simplest case when $\Gamma$ is a lattice, we feel that different exponential decay rates of the geometry along different cusps might cause trouble for the measure $\mu^{\sigma}$ to be finite. But if we assume the following control of global geometry:

$$
\lim _{R \rightarrow \infty} \frac{\operatorname{Vol} S(x, R)}{e^{h R}}=c(x),
$$

for a point $x \in H$ and for a positive number $c(x)$ (this is true if $H$ has a cocompact quotient), then we have

Theorem F. Let $\Gamma$ be a lattice satisfying $(*)$. Then the following is true:

(1) $\mu^{\sigma}(S M)<\infty$.

If moreover $(*)$ is true for all $x$ and if $c(x)$ is a $L^{1}$ function on $M$, then we have:

(2) $h^{2}=\int_{M}\left(\int_{S_{x} M}\left(R^{H}(v)+\operatorname{Ricci}(v)-R(v(0))\right) d \sigma_{x}(v)\right) d x$, where $d x$ is the Riemannian volume (normalized such that $\int_{S M} d \sigma_{x} d x=1$ ), $R^{H}$ is the scalar curvature of the horosphere, Ricci denotes the Ricci curvature on $S M$ and $R$ is the scalar curvature on $M$.

(3) If $\operatorname{dim} M=3$, then $h^{2}=\int_{M}\left[\int_{S_{x} M}(\operatorname{Ricci}(v)-R(v(0))) d \sigma_{x}(v)\right] d x$. If $\operatorname{dim} M=$ 2 , then $h^{2}=\int_{M}-c(x) K(x) d x / \int_{M} c(x) d x$, where $K(x)$ is the sectional curvature at $x$.

Next we consider the case when $\operatorname{dim} M=2$. Assuming that $(*)$ is true, then $d \omega^{s s} \triangleq d x d \sigma_{x}$ gives an invariant measure of the contracting horocycle flow $h^{t}$ with the natural parametrization (i.e. the induced Riemannian length along the horocycle foliation). By the same arguments as in [Yu1], $d \omega^{s s}$ is generically not invariant under the geodesic flow $g^{t}$ and it is invariant under $g^{t}$ if and only if $M$ has constant curvature. However, one can reparametrize the horocycle flow $h^{t}$ such that the measure $\mu^{\sigma}$ in Theorem F (1) is invariant under $h^{t}$ (see [M1]). The following theorem partially answers a question raised by J. Feldman and M. Ratner on the existence of a uniform parametrization of the horocycle flow on a finite volume surface of variable negative curvature and the corresponding Raghunathan's conjecture.

Theorem G. Let $M$ be a finite volume surface with negative curvature $-K_{2}^{2} \leq$ $K \leq-K_{1}^{2}, K_{2} \geq K_{1}>0$. Assuming that $(*)$ is true for some point $x$ in the universal cover, then we have

(1) There is a uniform parametrization $\mu^{\sigma}$ of the horocycle flow with finite total mass.

(2) (Orbit closures for horocycle flows; this is true without the extra assumption $(*)$ ). For each $v \in S M$, either $\overline{h^{t} v}=S M$ or $h^{t} v=\overline{h^{t} v}$ is periodic.

(3) (Classification of invariant measures). Let $\mu$ be any ergodic $h^{t}$-invariant Borel probability measure on $S M$. Then either $\mu=c \cdot \mu^{\sigma}$ (c is a constant) or $\mu$ is supported on a periodic orbit of $h^{t}$. 
(4) (Uniform distibution of horocycle orbits). If $v \in S M$ and $h^{t} v$ is not a periodic orbit, then for every bounded continuous function $f$ on $S M$ we have

$$
\lim _{t \rightarrow \infty} \frac{1}{t} \int_{0}^{t} f\left(h^{s} v\right) d s=\int_{S M} f d \mu^{\sigma} / \mu^{\sigma}(S M) .
$$

For a finite volume Riemannian manifold of pinched negative curvature, it is not hard to see that its geodesic flow is ergodic with respect to the finite Liouville measure. Similarly, we have the following theorem concerning the harmonic measure.

Theorem H. Let $\Gamma$ be a lattice. Let $\sigma_{x}$ be the harmonic measure on $\partial H$ and $\nu^{\sigma}$ be the measure on $S M$ as constructed in Proposition 5.2.2. Then the geodesic flow is ergodic with respect to $\nu^{\sigma}$.

(0.6) Connections between $\delta(\Gamma)$ and $\lambda(\Gamma)$. If $H$ is a symmetric space of real rank 1 , then according to Sullivan $([\mathrm{S} 1])$ and Corlette $([\mathrm{Co}])$, the bottom spectrum $\lambda(\Gamma)$ of the Laplacian satisfies

$$
\lambda(\Gamma)= \begin{cases}\frac{1}{4} h^{2} & \text { if } \delta(\Gamma) \leq \frac{h}{2}, \\ \delta(\Gamma)(h-\delta(\Gamma)) & \text { if } \delta(\Gamma) \geq \frac{h}{2}\end{cases}
$$

where $h=\lim _{R \rightarrow \infty} \frac{\log \operatorname{Vol} S(x, R)}{R}$. For the general variable curvature case, there is definitely no such simple relation. However, the above result is still true if one assumes that $H$ is harmonic with a compact quotient (and it is generally believed that such an $H$ must be symmetric).

(0.7) Rigidity. Finally, we address some rigidity problems. Assume that the Poincaré series of $\Gamma_{1}$ diverges at $\delta\left(\Gamma_{1}\right)$. For any homomorphism $\Gamma_{1} \stackrel{\varphi}{\longrightarrow} \Gamma_{2}$, there always exists a $\varphi$-equivariant map $L\left(\Gamma_{1}\right) \stackrel{\Phi}{\longrightarrow} L\left(\Gamma_{2}\right)$ measurable with respect to the Patterson-Sullivan measures (see [Yu4] for more discussions about the existence). Assuming that a measurable $\Phi^{-1}$ exists, then we have

Theorem I. Under the above assumptions, we have

(1) Either $\Phi^{-1}$ is singular with respect to the conformal densities or $\Phi$ preserves the Busemann cross ratio up to a homothetic change of the Riemannian metric.

(2) In the latter case, $\Phi$ is conformal with respect to the Busemann metric and there is a measurable time-preserving conjugacy between the geodesic flows on $\Omega\left(\Gamma_{1}\right)$ and $\Omega\left(\Gamma_{2}\right)$.

(3) If moreover, $\Gamma_{1}$ and $\Gamma_{2}$ are convex cocompact, then any isomorphism between them always induces a homeomorphism between their limit sets which conjugates their actions on the limit sets. In particular, the following properties are equivalent:

a) $\delta\left(\Gamma_{1}\right)=\delta\left(\Gamma_{2}\right)$ and $\Phi$ is nonsingular with respect to the conformal densities.

b) $\Phi$ preserves the cross ratio.

c) $\delta\left(\Gamma_{1}\right)=\delta\left(\Gamma_{2}\right)$ and $\Phi$ is conformal with respect to the Busemann metric.

d) $M_{1}=H_{1} / \Gamma_{1}$ and $M_{2}=H_{2} / \Gamma_{2}$ have the same marked length spectrum.

e) There exists a continuous time-preserving conjugacy of the geodesic flows on $\Omega\left(\Gamma_{1}\right)$ and $\Omega\left(\Gamma_{2}\right)$. 
Note. Various aspects of Theorem A were proved by Hopf (1939, for hyperbolic 2 -space) and D. Sullivan (1979-1982, for hyperbolic $n$-space). The completely conservative or completely dissipative dichotomy for the geodesic flow was first observed by Poincaré (1906, for Riemann surface) and extended by Sullivan to the $n$-dimensional hyperbolic space. For the compact variable curvature case, the geodesic flow is extensively studied under the guise of hyperbolic dynamics by Anosov, Sinai, Smale... since the early sixties. S. J. Patterson and D. Sullivan were among the earliest (since the late seventies) to study the ergodic theory of general noncocompact discrete subgroups in $S O(n, 1)$. Later on (1990), K. Corlette generalized Sullivan's work to convex cocompact groups in other noncompact rank 1 Lie groups and discovered striking rigidity phenomena.

A first version of this paper appeared in the spring of 1992 as an MSRI preprint. Since then, I had been trying to prove some rigidity results for symmetric spaces which were first intended to be part of section 7 in the present paper but, due to its length, will appear separately (see [Yu3] [Yu4]). Recently I received two preprints (V. A. Kaimanovich, Ergodic theory of harmonic measures for the geodesic flow on hyperbolic spaces, 1993; and K. Corlette, A. Iozzi, Limit sets of isometry groups of exotic hyperbolic spaces) which are related to our work. The three papers have intersections, but do not subsume the results of the others. The results in section 6 of the present paper generalize some of Corlette's work on convex-cocompact groups of symmetric spaces of real rank 1 (see $[\mathrm{Co}]$ ). Recall that convex-cocompact groups are geometrically finite groups with no cusps. In the paper of Corlette-Iozzi, the authors study the ergodic theory of geometrically finite groups of symmetric spaces of real rank 1, and obtain results analogous to the results for convex-cocompct groups in $[\mathrm{Co}]$ and the present paper. As we pointed out in section (0.5), for geometrically finite groups in the variable curvature case, different asymptotic geometric behaviors along different cusps might cause serious trouble. Even for a lattice, the simplest geometrically finite group, the ergodicity and uniqueness problem of its Patterson-Sullivan measure is still open. Our Theorem F and Theorem G are partial results along this direction. In a different direction, the paper of Kaimanovich studies the Hopf dichotomy for a harmonic measure associated with a Markov operator on a Gromov hyperbolic space. Kaimanovich's result is analogous to our Theorem A or Theorem B, but does not overlap with ours. As he remarked in page 57 of his paper, it would be interesting to obtain both his result and ours in a unified manner.

There is already a rich and beautiful ergodic theory for discrete groups in $S O(n, 1)$ and a even richer theory for Kleinian groups. The discrete groups of other exotic (the complex, the quaternionic and the Cayley) hyperbolic spaces tends to be more rigid. Our main motivation in studying the general variable curvature case is to pave the way for the study of various rigidity problems concerning discrete subgroups in non-compact semi-simple Lie groups of real rank 1, and possible extensions of the Mostow rigidity to discrete groups of infinite covolume. Section 7 lays the foundation for further study. The sequels [Yu3], [Yu4] represents, more or less, the completion of our program.

\section{Gromov's metric AND three other metrics at infinity}

(1.1) Notations and definitions, Gromov's metric. Throughout this paper we consider a simply connected Riemannian manifold $H$ with bounded negative 
curvature $\infty<-K_{2}^{2} \leq K \leq K_{1}^{2}<0$. As a metric space with respect to the Riemannian metric $d$, it is geodesic and hyperbolic in the sense of Gromov $([\mathrm{G}])$. Fix a base point $x_{0} \in H$ and consider the Gromov product

$$
(x \cdot y)_{x_{0}} \triangleq \frac{1}{2}\left(d\left(x, x_{0}\right)+d\left(y, x_{0}\right)-d(x, y)\right) .
$$

A sequence of points $\left(x_{n}\right)$ in $H$ is said to be converging to infinity if $\left(x_{n} \cdot x_{m}\right)_{x_{0}} \rightarrow \infty$ as $n, m \rightarrow \infty$. Two such sequences $\left(x_{n}\right)$ and $\left(y_{n}\right)$ are equivalent if and only if $\left(x_{n} \cdot y_{n}\right)_{x_{0}} \rightarrow \infty$ as $n \rightarrow \infty$. The set of all such equivalence classes is defined to be the ideal boundary of $H$ and is denoted by $\partial H$.

In order to study the asymptotic geometry of $\partial H$, Gromov introduced the following metric on the boundary. Let $\epsilon>0$ be a positive number. For each curve $\sigma:\left[t_{1}, t_{2}\right] \rightarrow H$ define the $\epsilon$-length

$$
\ell_{\epsilon}(\sigma)=\int_{t_{1}}^{t_{2}} e^{-\epsilon d\left(x_{0}, \sigma(t)\right)} d t
$$

For any two points $x, y \in H$, the Gromov metric is defined to be (the infimum is taken over all continuous curves connecting $x, y$ )

$$
|x-y|_{x_{0}}^{\epsilon}=\inf _{\sigma} \ell_{\epsilon}(\sigma)
$$

There exists a constant $\epsilon_{0}=\epsilon_{0}\left(K_{2}\right)>0$ such that for all $0<\epsilon<\epsilon_{0}$ the Gromov metric is a distance and moreover one has(see for example $[G]$ and the references there):

(i) The identity map $(H, d) \leftrightarrow\left(H,|\cdot|{ }_{x_{0}}^{\epsilon}\right)$ extends continuously to a homeomorphism $\bar{H} \leftrightarrow \bar{H}_{\epsilon}$, where $\bar{H}=H \cup \partial H$ and $\bar{H}_{\epsilon}$ is the metric completion of $\left(H,|\cdot| \begin{array}{l}\epsilon \\ x_{0}\end{array}\right)$. Thus in particular, $|\cdot|_{x_{0}}^{\epsilon}$ defines a metric on $\partial H$.

(ii) Every isometry $\gamma$ of $H$ induces a $|\cdot|{ }_{x_{0}}^{\epsilon}$-Lipschitz map on $\left(\partial H,|\cdot|{ }_{x_{0}}^{\epsilon}\right)$. For each $\zeta \in \partial H$, there exists a neighborhood $U \ni \zeta$ and a constant $C=C\left(K_{1}, \epsilon\right)$ so that for all $y_{1}, y_{2} \in U$,

$$
C^{-1} e^{\epsilon \rho_{x_{0}, \zeta}\left(\gamma^{-1} x_{0}\right)} \leq \frac{\left|\gamma y_{1}-\gamma y_{2}\right|_{x_{0}}^{\epsilon}}{\left|y_{1}-y_{2}\right|_{x_{0}}^{\epsilon}} \leq C e^{\epsilon \rho_{x_{0}, \zeta}\left(\gamma^{-1} x_{0}\right)}
$$

where $\rho_{x, \zeta}(y)$ is the Busemann function at $\zeta$ with $\rho_{x, \zeta}(x)=0$.

(1.2) Three other metrics. We introduce three other metrics at infinity suggested by Kaimanovich $([\mathrm{K}])$. They are all equivalent to the Gromov metric. In many of our later arguments, some prefer one particular metric, some prefer another. We will exploit them correspondingly.

The shadow metric. Fix a point $x_{0} \in H$ and for $\zeta, \eta \in \partial H$ define

$$
\begin{aligned}
& \ell_{x_{0}}(\zeta, \eta) \triangleq \sup \left\{t \mid t \geq 0, \quad d\left(\gamma_{x, \eta}(t), \gamma_{x, \eta}(t)\right) \leq 1\right\} \\
& \rho_{x_{0}}^{\epsilon}(\zeta, \eta) \triangleq e^{-\epsilon \ell_{x_{0}}(\zeta, \eta)}
\end{aligned}
$$

where $\gamma_{x, \zeta}(t)$ and $\gamma_{x, \eta}(t)$ are the geodesics starting from $x$ and pointing to $\zeta, \eta$. This metric is called the shadow metric. 
(1.2.2) The Busemann metric. Consider the Busemann cocycle $\beta_{x}(\zeta, \eta) \triangleq \rho_{x, \zeta}(y)+$ $\rho_{x, \eta}(y)$ where $y$ belongs to the geodesic $\gamma$ from $\zeta$ to $\eta$. It is easy to see that the definition does not depend on the choice of $y$ and geometrically, $\beta_{x}(\zeta, \eta)$ is the length of the segment on $\gamma$ cut out by the horospheres passing through $x$ and centered at $\zeta, \eta$. The Busemann metric is defined to be

$$
B_{x_{0}}^{\epsilon}(\zeta, \eta) \triangleq e^{-\frac{1}{2} \epsilon \beta_{x_{0}}(\zeta, \eta)}
$$

(1.2.3) The geodesic metric. For any two points $\zeta, \eta \in \partial H$ consider the geodesic $\gamma$ from $\zeta$ to $\eta$ and let $D_{x_{0}}(\zeta, \eta)$ be the distance from $x_{0}$ to $\gamma$. The geodesic metric is defined to be

$$
d_{x_{0}}^{\epsilon}(\zeta, \eta) \triangleq e^{-\epsilon D_{x_{0}}(\zeta, \eta)} .
$$

Proposition (1.2.1) ([C], $[\mathrm{K}])$. There exist $\epsilon_{0}=\epsilon_{0}\left(K_{1}\right)>0$ and $C=C\left(K_{1}, K_{2}\right)$ $>0$ such that $\rho_{x_{0}}^{\epsilon}(\cdot), B_{x_{0}}^{\epsilon}(\cdot), d_{x_{0}}^{\epsilon}(\cdot)$ are distances for all $0<\epsilon \leq \epsilon_{0}$. Moreover, for all $x_{0} \in H$ and $\zeta, \eta \in \partial H$ and for any two dist $_{x_{0}}^{\epsilon, 1}$, dist ${ }_{x_{0}}^{\epsilon, 2}$ of the above four metrics

$$
C^{-1} \leq \frac{\operatorname{dist}_{x_{0}}^{\epsilon, 1}(\zeta, \eta)}{\operatorname{dist}_{x_{0}}^{\epsilon, 2}(\zeta, \eta)} \leq C
$$

Among the four metrics defined above, there is a special one: the Busemann metric $B_{x_{0}}^{\epsilon}$. All isometries of $H$ act on $\left(\partial H, B_{x_{0}}^{\epsilon}\right)$ as conformal maps under this metric.

Proposition (1.2.2). Each isometry $\gamma$ on $H$ induces a conformal map on $\partial H$ under the metric $B_{x_{0}}^{\epsilon}$.

Proof. Given any two points $\zeta, \eta \in \partial H$, it is easy to see that

$$
B_{x_{0}}^{\epsilon}(\gamma \zeta, \gamma \eta)=e^{-\frac{1}{2} \epsilon\left[\rho_{x_{0}, \gamma \zeta}\left(\gamma x_{0}\right)+\rho_{x_{0}, \gamma \eta}\left(\gamma x_{0}\right)\right]} B_{x_{0}}^{\epsilon}(\zeta, \eta) .
$$

For each $\delta>0$ there is a neighborhood $V$ of $\zeta$ in $\partial H$ such that for all $\eta \in V$, we have

$$
\left.1-\delta \leq e^{\frac{\epsilon}{2}\left[\rho_{x_{0}, \gamma \zeta}{ }^{\left(\gamma x_{0}\right)}+\rho_{x_{0}, \gamma \eta}\left(\gamma x_{0}\right)\right.}\right] / e^{-\epsilon \rho_{x_{0}, \gamma \zeta}\left(\gamma x_{0}\right)} \leq 1+\delta .
$$

Thus for all $\eta \in V$ we have $(1-\delta) e^{-\epsilon \rho_{x_{0}, \gamma \zeta}\left(\gamma x_{0}\right)} \leq \frac{B_{x_{0}}^{\epsilon}(\gamma \zeta, \gamma \eta)}{B_{x_{0}}^{\epsilon}(\zeta, \eta)} \leq(1+\delta) e^{-\epsilon \rho_{x_{0}, \gamma \zeta}\left(\gamma x_{0}\right)}$ and the proposition follows.

\section{The aCtion of $\Gamma$ on $\partial H$, QUasi-invariant measures}

(2.1) The topological trichotomy ([E-O'N]). Let $\Gamma$ be a torsion free discrete isometry group acting on $H$ freely and properly discontinuously. For any point $x$ in $H$ consider the orbit $\Gamma x$ and its closure $\overline{\Gamma x}$ under the Gromov metric. The limit set of $\Gamma$ is defined by $L(\Gamma) \triangleq \overline{\Gamma x} \cap \partial H$. According to Eberlein and O'Neil, one of the following possibilities must occur (the topological trichotomy):

(i) $L(\Gamma)$ is a singleton. Every element of $\Gamma$ is parabolic with a common fixed point.

(ii) $L(\Gamma)$ consists of two points $\zeta, \eta$. $\Gamma$ is infinite cyclic and every element of $\Gamma$ is hyperbolic with the common axis $\gamma_{(\zeta, \eta)}$.

(iii) $L(\Gamma)$ is infinite.

We will call $\Gamma$ Fuchsian if its limit set satisfies (iii). From now on we always assume $\Gamma$ to be a Fuchsian group. 
(2.2) The action of $\Gamma$ on $\partial H$ (see $[G][C]$ ). The following theorem is due to Gromov.

Theorem (2.2.1). $\quad L(\Gamma)$ is the unique $\Gamma$-invariant minimal closed set of $\partial H$.

As corollaries of Gromov's theorem, we have:

(i) The $\Gamma$-orbit of each $\zeta \in L(\Gamma)$ is dense in $L(\Gamma)$.

(ii) $\mathrm{Fix}^{h} \triangleq\{$ fixed points of hyperbolic elements in $\Gamma\}$ is dense in $L(\Gamma)$.

(iii) $\operatorname{Fix}^{p} \triangleq\{$ fixed points of parabolic elements in $\Gamma\}$ is either empty, or dense in $L(\Gamma)$.

(iv) $L(\Gamma)$ is a perfect subset of $\partial H$. Either $L(\Gamma)=\partial H$ or $L(\Gamma)$ is nowhere dense in $\partial H$.

We define the ordinary set of $\Gamma$ by $O(\Gamma)=\partial H \backslash L(\Gamma)$. It is well-known that the action of $\Gamma$ on $H \cup O(\Gamma)$ is properly discontinuous ([E-O'N]). The Kleinian manifold associated to $\Gamma$ is defined to be $\bar{M}=(H \cup O(\Gamma)) / \Gamma$. The following definition is extremely useful when studying the ergodic properties of $\Gamma$.

Definition (2.2.2). The radial limit set $L^{r}(\Gamma)$ is the set of all $\zeta \in L(\Gamma)$ such that any geodesic ray joining $x \in H$ and $\zeta$ intersects some $\epsilon$-neighborhood of $\Gamma x$ infinitely many times.

Obviously $L^{r}(\Gamma)$ is non-empty and hence it is dense in $L(\Gamma)$ by (i).

(2.3) Quasi-invariant measures of $\Gamma$ on $\partial H$. Our objective in this paper is to study the ergodic theory of Fuchsian groups, in connection with the geometry of the Fuchsian manifold $M=H / \Gamma$ and the dynamics of the geodesic flow. The following proposition clarifies one fundamental fact.

Proposition (2.3.1). There is no finite invariant measure for the action of $\Gamma$ on $L(\Gamma)$.

Proof. Let $\gamma$ be a hyperbolic element in $\Gamma$ with fix points $\gamma^{-}, \gamma^{+}$(its existence follows from the above discussion). Then any finite $\Gamma$-invariant measure $\mu$ is first of all $\gamma$-invariant and by the hyperbolicity of $\gamma, \mu$ must be supported on $\gamma^{-}, \gamma^{+}$. The orbits $\Gamma \gamma^{-}$and $\Gamma \gamma^{+}$are both infinite $\Rightarrow \mu$ is infinite. This is a contradiction.

This proposition tells us that instead of invariant measures, we should look for special quasi-invariant measures which are related to the geometry of the manifold and the action of the group. Therefore we introduce the following definition:

Definition (2.3.2). A family of finite Borel measures $\left\{m_{x}\right\}_{x \in H}$ on $\partial H$ is called an $f$-density of $\Gamma$ for a measurable function $f: H \times H \times \partial H \rightarrow \mathbb{R}$ if for all points $x, y \in H$ the measures $m_{x}, m_{y}$ are equivalent with Radon-Nikodym derivatives

$$
\frac{d m_{y}}{d m_{x}}(\zeta)=f(x, y, \zeta)
$$

for $m_{x}$-a.e. $\zeta \in \partial H$ and for all $\gamma \in \Gamma, f\left(\gamma^{-1} x, x, \gamma^{-1} \zeta\right)=f(x, \gamma x, \zeta)$.

Notice that the last condition is equivalent to that $m_{x}(A)=m_{\gamma x}(\gamma A)$ for all measurable sets $A \subset \partial H$. The following definition introduces probably the two most important quasi-invariant measures.

Definition (2.3.3). (i) Let $K_{\lambda}(x, y, \zeta)$ be the $\lambda$-Poisson kernel of $H$ (see (4.1)). If in Definition (2.3.2) $f(x, y, \zeta)=K_{\lambda}(x, y, \zeta)$ then the corresponding $K_{\lambda}$-density is called a $\lambda$-harmonic density of $\Gamma$. 
(ii) If $f(x, y, \zeta)=e^{-\alpha \rho_{x, \zeta}(y)}$ then the corresponding $f$-density is called an $\alpha$ conformal density of $\Gamma$.

(iii) A finite Borel measure $m$ on $L(\Gamma)$ is called an $\alpha$-quasi-conformal density of $\Gamma$ if there exists some constant $C \geq 1$ such that for some $x_{0} \in H$ and for all $\gamma \in \Gamma$

$$
C^{-1} e^{-\alpha \rho_{\gamma^{-1} x_{0}, \zeta^{\left(x_{0}\right)}}} \leq \frac{d\left(\gamma^{*} m\right)}{d m}(\zeta) \leq C e^{-\alpha \rho_{\gamma^{-1} x_{0}, \zeta^{\left(x_{0}\right)}}} .
$$

Remark. If $\left\{m_{x}\right\}_{x \in H}$ is an $\alpha$-conformal density, then $m_{x_{0}}$ obviously gives rise to a quasi-conformal density.

\section{Conformal Densities}

(3.1) Conformal densities arising from Hausdorff measure. Let $(X, \rho)$ be any metric space and $D \geq 0$ be a nonnegative constant. Let $A$ be a subset of $X$. For each $\epsilon \geq 0$ consider

$$
\mathcal{H}_{\epsilon}^{D}(A) \triangleq \inf \left\{\sum_{j=1}^{\infty} \delta_{j}^{D} \mid A \subset \bigcup_{j} B_{x_{j}}\left(\delta_{j}\right), \delta_{j} \leq \epsilon, x_{j} \in A\right\}
$$

where the infimum is taken among all coverings of $A$ by balls of radius $\leq \epsilon$. The limit measure $\mathcal{H}_{\rho}^{D}(A)=\lim _{\epsilon \rightarrow 0} \mathcal{H}_{\epsilon}^{D}(A)$ is called the $D$-dimensional Hausdorff measure of $A$. The Hausdorff dimension $H D(A)$ is defined to be

$$
H D(A) \triangleq \inf \left\{D: \mathcal{H}_{\rho}^{D}(A)=0\right\}=\sup \left\{D: \mathcal{H}_{\rho}^{D}(A)=\infty\right\} .
$$

An easy consequence of this definition is that if $0<\mathcal{H}_{\rho}^{D}(A)<\infty$ then $H D(A)=D$. The following statement follows easily from the above definition, Proposition (1.2.1) and Proposition (1.2.2). Here the Busemann metric is singled out as the favorite among the four metrics constructed in section 1.

Proposition (3.1.1). Suppose $A \subset \partial H$ is an $\Gamma$-invariant Borel set with $\mathcal{H}_{\rho}^{D / \epsilon}(A)$ $<\infty$ where $\rho$ is any of the four metrics $|\cdot|_{x_{0}}^{\epsilon}, \rho_{x_{0}}^{\epsilon}, d_{x_{0}}^{\epsilon}, B_{x_{0}}^{\epsilon}$. Then $\mathcal{H}_{\rho}^{D / \epsilon}$ defines a $D$ quasi-conformal density of $\Gamma$ on $\partial H$. In particular, $\left\{\mathcal{H}_{B_{x_{0}}^{D / \epsilon}}^{D / \epsilon}\right\}_{x_{0} \in H}$ is a D-conformal density of $\Gamma$.

Later on we will see that in many cases Hausdorff measure is the only way to produce conformal density.

(3.2) The Patterson-Sullivan construction. Next we generalize Patterson's construction of an important conformal density for any Fuchsian group $\Gamma$ acting on a manifold of negative curvature. Fix two points $x, y$ in $H$ and for any real number $s>0$, consider the Poincaré series

$$
g_{s}(x, y)=\sum_{\gamma \in \Gamma} e^{-s d(x, \gamma y)}
$$

Let $S_{k}$ be the number of the orbit points $\Gamma y$ in $B\left(x, k+\frac{1}{2}\right) \backslash B\left(x, k-\frac{1}{2}\right)$; then $g_{s}(x, y)$ is proportional to $\sum_{k=0}^{\infty} S_{k} e^{-k s}$. Let $\delta(\Gamma)$ be the critical exponent of $\Gamma$ defined 
by $\delta(\Gamma) \triangleq \varlimsup_{k \rightarrow \infty} \frac{1}{k} \log S_{k}$. Then $g_{s}(x, y)$ diverges for $s<\delta(\Gamma)$ and converges for $s>\delta(\Gamma)$. Consider the family of measures

$$
\mu_{x}^{s}=\frac{1}{g_{s}(y, y)} \sum_{\gamma \in \Gamma} e^{-s d(x, \gamma y)} \delta_{\gamma y}, \quad s<\delta(\Gamma)
$$

where $\delta(\gamma y)$ is the Dirac mass at $\gamma y$. Using the triangle inequality it is easy to see that $e^{-s d(x, y)} g_{s}(y, y) \leq g_{s}(x, y) \leq e^{s d(x, y)} g_{s}(y, y)$. Thus $\left\{\mu_{x}^{s}\right\}_{0 \leq s<\delta(\Gamma)}$ is a family of finite measures with uniformly bounded total mass. Let $\mu_{x}=\lim _{s_{j} \rightarrow \delta(\Gamma)+} \mu_{x}^{s_{j}}$ be a weak limit in the space of uniformly bounded measures on $H \cup \partial H$. Suppose that $g_{s}(y, y) \rightarrow \infty$ as $s \rightarrow \delta(\Gamma)$ (this restriction can be eliminated by adding a slowly increasing weight as in [P] [S1]). Then $\mu_{x}$ is concentrated on the cluster points of the orbit $\Gamma y$,i.e., the limit set $L(\Gamma)$. From the construction it is easy to see that for any other point $x^{\prime} \in H$ the limit $\lim _{s_{j} \rightarrow \delta(\Gamma)+} \mu_{x^{\prime}}^{s_{j}}=\mu_{x^{\prime}}$ also exists and moreover, the Radon-Nikodym derivative at $\zeta \in L(\Gamma)$ satisfies

$$
\frac{d \mu_{x^{\prime}}}{d \mu_{x}}(\zeta)=e^{-\delta(\Gamma) \rho_{x, \zeta}\left(x^{\prime}\right)} .
$$

It is also easy to see that $\gamma^{*} \mu_{x}=\mu_{\gamma x}$. So we have proved part (i) of the following proposition.

Proposition (3.2.1). $\quad$ (i) For any Fuchsian group $\Gamma$, there exists a $\delta(\Gamma)$-conformal density.

(ii) $\delta(\Gamma)>0$.

(iii) Suppose $\Gamma_{1} \subset \Gamma_{2} \subset \cdots \subset \Gamma=\cup \Gamma_{i}$ are subgroups of $\Gamma$. Then $\delta(\Gamma)=$ $\lim _{i \rightarrow \infty} \delta\left(\Gamma_{i}\right)$.

Part (ii) is equivalent to Proposition (2.3.1). The proof of (iii) goes exactly like the proof in Sullivan([S1]).

(3.3) Uniqueness. The weak limit in Patterson's construction is by no means unique. The following statement reveals that uniqueness is closely related to ergodicity.

Proposition (3.3.1). Let $\left\{\mu_{x}\right\}_{x \in H}$ be any $\alpha$-conformal density of $\Gamma$. Then any other $\alpha$-conformal density $\left\{\nu_{x}\right\}_{x \in H}$ coincides with $\mu$ up to a scalar multiplication if and only if the $\Gamma$ action on $\partial H$ is ergodic with respect to the measure class defined by $\mu$.

Proof. 1) If $\Gamma$ is ergodic with respect to the measure class of $\mu$, consider the measure $\sigma=\frac{1}{2}(\mu+\nu)$ which is clearly also an $\alpha$-conformal density. Since $\mu_{x}$ and $\nu_{x}$ are both absolutely continuous with respect to $\sigma_{x}$ therefore their RadonNikodym derivatives $\frac{d \mu_{x}}{d \sigma_{x}}, \frac{d \nu_{x}}{d \sigma_{x}}$ exist and are $\Gamma$-invariant. By the ergodicity of $\Gamma$ these derivatives are equal to positive constants $\mu_{x}$-almost everywhere.

2) If $\Gamma$ is not ergodic with respect to the measure class of $\mu$, then there exists a Borel $\Gamma$-invariant subset $A \subset \partial H$ such that for all $x \in H, \mu_{x}(A)>0$ and $\mu_{x}\left(A^{C}\right)>0$. Define $\sigma_{x}(E)=\mu_{x}(E \cap A)$; then it is easy to check that $\sigma_{x}$ is another $\alpha$-conformal density. 
(3.4) Sullivan's shadow lemma. Fix a point $x_{0}$ in $H$. For any $x \in H$ and $d>0$ consider the shadow of the ball $B(x, d)$ from $x_{0}$ to $\partial H$ defined by $O_{x_{0}}(x, d)=$ $\left\{\zeta \in \partial H \mid \gamma_{x_{0}, \zeta} \cap B(x, d) \neq \varnothing\right\}$, where $\gamma_{x_{o}, \zeta}$ is the geodesic from $x_{0}$ to $\zeta$. The following important result(in the constant curvature case) is due to Sullivan.

Lemma (3.4.1) (Sullivan's shadow lemma). Let $\left\{\mu_{x}\right\}_{x \in H}$ be an $\alpha$-conformal density of $\Gamma$. Suppose $\mu_{x_{0}}$ does not consist of a single atom. Then there exist constants $c \geq 1$ and $d_{0} \geq 0$, such that for all $d \geq d_{0}$ and $\gamma \in \Gamma$ one has

$$
c^{-1} e^{-\alpha d\left(x_{0}, \gamma^{-1} x_{0}\right)} \leq \mu_{x_{0}}\left(O_{x_{0}}\left(\gamma^{-1} x_{0}, d\right)\right) \leq c e^{-\alpha d\left(x_{0}, \gamma^{-1} x_{0}\right)+2 d \alpha} .
$$

Proof. This lemma is actually proved in [C] in the case of Gromov's hyperbolic space. For the sake of completeness, we give a simplified proof in our case. The geodesic distance $d_{x_{0}}^{\epsilon}$ turns out to be favorable for the argument here. Let $m_{0}$ be the largest mass of atoms of $\mu_{x_{0}}$ and fix any number $m$ such that $m_{0}<m<A \triangleq$ $\mu_{x_{0}}(\partial H)$. Then there exists $\delta>0$ such that all sets in $\partial H$ with $d_{x_{0}}^{\epsilon}$-diameter $\leq \delta$ have $\mu_{x_{0}}$-measure $\leq m$. From the definition of $d_{x_{0}}^{\epsilon}$ it is easy to see that there exists $d_{0}>0$ such that for all $\gamma \in \Gamma$ and $d \geq d_{0}$, the $d_{x_{0}}^{\epsilon}$-diameter of the set $\gamma\left(\partial H \backslash O_{x_{0}}\left(g^{-1} x_{0}, d\right)\right)$ is $\leq \delta$. Therefore we have $A-m \leq \mu_{x_{0}}\left(\gamma O_{x_{o}}\left(\gamma^{-1} x_{0}, d\right)\right) \leq A$. On the other hand,

$$
\begin{aligned}
\mu_{x_{0}}\left(\gamma O_{x_{0}}\left(\gamma^{-1} x_{0}, d\right)\right) & =\mu_{\gamma^{-1} x_{0}}\left(O_{x_{0}}\left(\gamma^{-1} x_{0}, d\right)\right) \\
& =\int_{O_{x_{0}}\left(\gamma^{-1} x_{0}, d\right)} e^{-\alpha \rho_{\gamma^{-1} x_{0}, \zeta^{\left(x_{0}\right)}}} d \mu_{x_{0}}(\zeta)
\end{aligned}
$$

By the property of the Busemann function $\rho_{x, \zeta}(y)$ it is easy to see that (compare also $[\mathrm{C}])$ for all $\zeta \in O_{x_{0}}\left(\gamma^{-1} x_{0}, d\right)$,

$$
d\left(x_{0}, \gamma^{-1} x_{0}\right)-d \leq \rho_{\gamma^{-1} x_{0}, \zeta}\left(x_{0}\right) \leq d\left(x_{0}, \gamma^{-1} x_{0}\right) .
$$

Combining the above estimates we obtain the proof of the lemma.

Although the idea of this lemma is simple, it shall play a crucial role in later studies.

(3.5) The radial limit set. The radial limit set (see (2.2.2) for definition) was introduced by Hedlund in dimension 2 when he was studying horocyclic transitive points. It also has connection with Diophantine approximation. Here it is of crucial importance for our study of metric properties (ergodicity, Hausdorff dimension,invariant measure ...) of $\Gamma$. Note that $\zeta \in \partial H$ is a radial limit point if and only if for some $c>0$ and $x \in H, \zeta$ belongs to infinitely many shadows $O_{x}(\gamma x, c), \gamma \in \Gamma$.

Theorem (3.5.1). Let $\sigma$ be a D-conformal density of $\Gamma$. If $\sum_{\gamma \in \Gamma} e^{-D d\left(x_{0}, \gamma^{-1} x_{0}\right)}<$ $\infty$, then $\sigma_{x_{0}}\left(L^{r}(\Gamma)\right)=0$.

Proof. The idea of our proof is due to Sullivan [S1]. We can write $\Gamma=\left\{\gamma_{n}\right\}_{n=1,2, \cdots}$. Given any $\epsilon>0$ there exists $N$ such that $\sum_{n \geq N} e^{-D d\left(x_{0}, \gamma^{-1} x_{0}\right)}<\epsilon$. By Sullivan's shadow lemma, for $d \geq d_{0}$,

$$
\sum_{n \geq N} \sigma_{x_{0}}\left(O_{x_{0}}\left(\gamma_{n}^{-1} x_{0}, d\right)\right) \leq C e^{2 d D} \epsilon .
$$


But for $0<d \leq d_{0}$ we also have

$$
\sum_{n \geq N} \sigma_{x_{0}}\left(O_{x_{0}}\left(\gamma_{n}^{-1} x_{0}, d\right)\right) \leq \sum_{n \geq N} \sigma_{x_{0}}\left(O_{x_{0}}\left(\gamma_{n}^{-1} x_{0}, d_{0}\right)\right) \leq C e^{2 d_{0} D} \epsilon .
$$

Consequently $\sigma_{x_{0}}\left[\bigcap_{N \geq 1} \bigcup_{n>N} O_{x_{0}}\left(\gamma_{n}^{-1} x_{0}, d\right)\right]=0$ for all $d>0$. But as we have already remarked, $L^{r}(\Gamma)=\bigcup_{d>0}\left(\bigcap_{N \geq 1} \bigcup_{n>N} O_{x_{0}}\left(\gamma_{n}^{-1} x_{0}, d\right)\right)$, so the theorem follows from the above estimates.

We now begin to study the Hausdorff dimension of the radial limit set $L^{r}(\Gamma)$. Next we use the shadow metric $\rho \triangleq \rho_{x_{0}}^{1}$.

Theorem (3.5.2). Let $\sigma$ be a D-conformal density of $\Gamma$. Then there is a constant $C>0$ such that for all $A \subset L^{r}(\Gamma)$ with positive measure $\sigma_{x_{0}}(A)>0$, one has $\mathcal{H}_{\rho}^{D}(A) \leq C \sigma_{x_{0}}(A)$.

Proof. For $\sigma_{x_{0}}$-almost every point $\zeta \in A$ we have

$$
\lim _{\epsilon \rightarrow 0} \frac{\sigma_{x_{0}}(D(\zeta, \epsilon) \cap A)}{\sigma_{x_{0}}(D(\zeta, \epsilon))}=1
$$

where $D(\zeta, \epsilon) \triangleq\{\eta \in \partial H \mid \rho(\zeta, \eta) \leq \epsilon\}$. Given $\delta>0$ there is a subset $A^{\prime} \subset A$ and $\epsilon_{0}>0, d_{1}>0$ with $\sigma_{x_{0}}\left(A \backslash A^{\prime}\right)<\delta$ such that

(i) $\frac{\sigma_{x_{0}}(D(\zeta, \epsilon) \cap A)}{\sigma_{x_{0}}(D(\zeta, \epsilon))} \geq 1-\delta$ for all $0<\epsilon \leq \epsilon_{0}$ and $\zeta \in A^{\prime}$.

(ii) $A^{\prime} \subset \bigcap_{N \geq 1} \bigcup_{n>N} O_{x_{0}}\left(\gamma_{n}^{-1} x_{0}, d_{1}\right)\left(\Gamma=\left\{\gamma_{n}\right\}_{n \geq 1}\right.$ as in (3.5.1)).

Since every point in $A^{\prime}$ lies in infinitely many balls $O_{x_{0}}\left(\gamma_{n}^{-1} x_{0}, d_{1}\right)$, using a Vitali type argument, one can construct a union of balls $O_{x_{0}}\left(\gamma_{n_{k}}^{-1} x_{0}, d_{1}\right), k=1,2, \cdots$, which covers $\sigma$-almost all of $A^{\prime}$, such that their $\rho$-radius $r_{k}$ satisfies $r_{k}<\epsilon<\frac{\epsilon_{0}}{2}$ and that the center of $O(k)$ is outside the union $\bigcup_{j=1}^{k-1} O(j)$, where $O(k)$ is the ball in $\partial H$ with radius $2 r_{k}$ and with the same center as $O_{x_{0}}\left(\gamma_{n_{k}}^{-1} x_{0}, d_{1}\right)$. Hence the set $\Omega=\bigcup_{k \geq 1} O_{x_{0}}\left(\gamma_{n_{k}}^{-1} x_{0}, d_{1}\right)$ is a disjoint union and by the shadow lemma, there exists a constant $C_{1}$ such that

$$
\sum_{k \geq 1} e^{-D d\left(x_{0}, \gamma_{n_{k}}^{-1} x_{0}\right)} \leq C_{1} \sigma_{x_{0}}(\Omega) .
$$

By the definition of the shadow metric, there exists a constant $C_{2}$ such that for all $k, r_{k} \leq C_{2} e^{-d\left(x_{0}, \gamma_{n_{k}}^{-1} x_{0}\right)}$. Consequently we get

$$
\mathcal{H}_{\epsilon}^{D}\left(A^{\prime}\right) \leq \sum_{k} r_{k}^{D} \leq C_{2}^{D} \sum_{k} e^{-D d\left(x_{0}, \gamma_{n_{k}}^{-1} x_{0}\right)} \leq C_{2}^{D} C_{1} \sigma_{x_{0}}(\Omega) .
$$

Letting $\epsilon \rightarrow 0$ we obtain

$$
\mathcal{H}^{D}\left(A^{\prime}\right) \leq C_{2}^{D} C_{1} \sigma_{x_{0}}\left(A^{\prime}\right) / 1-\delta \leq \frac{C_{1} C_{2}^{D}}{1-\delta}\left(\sigma_{x_{0}}(A)-\delta\right) .
$$

Letting $\delta \rightarrow 0$ we get $\mathcal{H}^{D}(A) \leq C \cdot \sigma_{x_{0}}(A)$ for some constant $\mathrm{C}$ and the theorem follows.

Corollary (3.5.3). If there exists a D-conformal density $\sigma$ of $\Gamma$, then the Hausdorff dimension of the radial limit set satisfies $H D\left(L^{r}(\Gamma)\right) \leq D$. In particular, $H D\left(L^{r}(\Gamma)\right) \leq \delta(\Gamma)$. 
Proof. If $\sigma\left(L^{r}(\Gamma)\right)>0$ then the claim follows easily from Theorem (3.5.2). Even if $\sigma\left(L^{r}(\Gamma)\right)=0$, we can still construct a cover of $L^{r}(\Gamma)$ as in the proof of Theorem (3.5.2) and have $\mathcal{H}_{\epsilon}^{D}\left(L^{r}(\Gamma)\right) \leq C \sigma_{x}(\Omega)$ for some constant $C$. But this means that $\mathcal{H}^{D}\left(L^{r}(\Gamma)\right)<\infty$ and consequently, $H D\left(L^{r}(\Gamma)\right) \leq D$.

Proposition (3.5.4). A radial limit point $\zeta \in L^{r}(\Gamma)$ cannot be the atom of any $d$-conformal density $\mu$ of $\Gamma$.

Proof. Assume that $\zeta$ is both a radial point and a atom. Then there exist $\gamma_{i} \in$ $\Gamma, i \geq 1$, and $x \in H$ so that $\lim _{i \rightarrow \infty} e^{-\rho_{x, \zeta}\left(\gamma_{i} x\right)}=\infty$. If $\zeta$ is not a parabolic point, then we have:

$$
\sum_{\gamma \in \Gamma} e^{-\alpha \rho_{x, \zeta}(\gamma x)}=\sum_{\gamma \in \Gamma} \mu_{x}\left(\gamma^{-1} \zeta\right) / \mu_{x}(\zeta) \leq \frac{\mu_{x}(\partial H)}{\mu_{x}(\zeta)}<\infty,
$$

which is a contradiction. If $\zeta$ is a point both radial and parabolic, then the stabilizer $\Gamma_{\zeta}$ preserves all horospheres centered at $\zeta$. It follows that $\left\{\gamma_{i}\right\}$ contains no two elements from the same coset of $\Gamma / \Gamma_{\zeta}$. Yet $\mu_{x}(\partial H)<\infty$ implies $\sum e^{-\alpha \rho_{x, \zeta}(\gamma x)}<\infty$ over a system of cosets representatives of $\Gamma / \Gamma_{\zeta}$. This is again a contradiction.

The following theorem reveals an important property of the radial limit set.

Theorem (3.5.5). Let $\sigma$ be an $\alpha$-conformal density of $\Gamma$. If $A$ is a $\Gamma$-invariant subset of $L^{r}(\Gamma)$ then either $\sigma_{x}(A)=0$ or $\sigma_{x}(A)=\sigma_{x}(\partial H)$.

Proof. Suppose that $\sigma_{x}(A)>0$. For each density point $\zeta$ of $A$ there exist a number $C>0$ and a sequence $\left\{\gamma_{n}^{-1} x\right\}$ converging to $\zeta$ radially such that

$$
\frac{\sigma_{x}\left(O_{x}\left(\gamma_{n}^{-1} x, C\right) \cap A\right)}{\sigma_{x}\left(O_{x}\left(\gamma_{n}^{-1} x, C\right)\right)} \rightarrow 1(n \rightarrow \infty) .
$$

Let $m_{0}$ be the largest point mass of $\sigma_{x}$. Given $\epsilon>0$, if $C$ and $n$ are large enough, then we have

$$
\sigma_{\gamma_{n}^{-1} x}\left(O_{x}\left(\gamma_{n}^{-1} x, C\right)\right) \geq \sigma_{x}(\partial H)-m_{0}-\epsilon .
$$

Since $A$ is $\Gamma$-invariant, we have the following estimates:

$$
\begin{aligned}
& \frac{\sigma_{\gamma_{n}^{-1} x}\left(O_{x}\left(\gamma_{n}^{-1} x, C\right) \cap A\right)}{\sigma_{\gamma_{n}^{-1} x}\left(O_{x}\left(\gamma_{n}^{-1} x, C\right)\right)}=\frac{\sigma_{x}\left(O_{\gamma_{n} x}(x, C) \cap A\right)}{\sigma_{x}\left(O_{\gamma_{n} x}(x, C)\right)} \\
&= 1-\frac{\int_{O_{x}\left(\gamma_{n}^{-1} x, c\right) \backslash A} e^{-\alpha \rho_{x, \zeta}\left(\gamma_{n}^{-1} x\right)} d \sigma_{x}(\zeta)}{\int_{O_{x}\left(\gamma_{n}^{-1} x, c\right)} e^{-\alpha \rho_{x, \zeta}\left(\gamma_{n}^{-1} x\right)} d \sigma_{x}(\zeta)} \\
& \geq 1-C_{1} \frac{\sigma_{x}\left(O_{x}\left(\gamma_{n}^{-1} x, c\right) \backslash A\right)}{\sigma_{x}\left(O_{x}\left(\gamma_{n}^{-1} x, c\right)\right)} \text { (by the boundedness } \\
&\left.\quad \text { of the function } \rho_{x, \zeta}\left(\gamma_{n}^{-1} x\right)-\rho_{x, \eta}\left(\gamma_{n}^{-1} x\right) \text { for } \zeta, \eta \in O_{x}\left(\gamma_{n}^{-1} x, C\right)\right)
\end{aligned}
$$

$\geq 1-\varepsilon$ (by (1), if $n$ is large enough).

Combining (2), (3) we get, for $n$ large enough,

$$
\begin{aligned}
\sigma_{x}(A) & \geq \sigma_{x}\left(O_{\gamma_{n} x}(x, C) \cap A\right) \geq(1-\epsilon) \sigma_{\gamma_{n}^{-1} x}\left(O_{x}\left(\gamma_{n}^{-1} x, C\right)\right) \\
& \geq(1-\epsilon)\left(\sigma_{x}(\partial H)-m_{0}-\epsilon\right) .
\end{aligned}
$$


And consequently we get

$$
\sigma_{x}(A) \geq \sigma_{x}(\partial H)-m_{0} .
$$

But if $\sigma_{x}$ has any atom, then it has infinitely many and by (4) at least one of them lies in $A$. This is a contradiction to Proposition (3.5.4).

Corollary (3.5.6). Suppose that there exists an $\alpha$-conformal density of the Fuchsian group $\Gamma$ with $\sigma_{x}\left(L^{r}(\Gamma)\right)>0$. Then we have:

(i) $\sigma_{x}\left(L^{r}(\Gamma)\right)=\sigma_{x}(\partial H)$.

(ii) $\alpha=\delta(\Gamma)$.

(iii) $\alpha$ is the unique $\delta(\Gamma)$-conformal density of $\Gamma$ and $\Gamma$ is ergodic on $\partial H$ with respect to the measure class of $\sigma$.

(iv) $\Gamma$ is of divergence type.

Proof. (i) follows from Theorem (3.5.5) since $L^{r}(\Gamma)$ is $\Gamma$-invariant. (ii) follows from Corollary (3.5.3). (iii) is obvious. (iv) follows from Theorem (3.5.1).

We will see later on in section (5.3) that if $\Gamma$ is of divergence type, then the Patterson-Sullivan measure $\sigma_{x}$ satisfies $\sigma_{x}\left(L^{r}(\Gamma)\right)=\sigma_{x}(L(\Gamma))$.

(3.6) The lattice counting function. If $x, y \in H$ and $r>0$ set the lattice point counting function

$$
N(r, x, y)=\#\{\gamma \in \Gamma \mid d(x, \gamma y) \leq r\} .
$$

The following theorem will be important for later estimates.

Theorem (3.6.1). For any Fuchsian group $\Gamma$ there exists a constant $C=C(x, y)$ $>0$ so that $N(r, x, y) \leq C e^{\delta(\Gamma) r}$.

Proof. It is enough to prove $N(r, x, x) \leq C e^{\delta(\Gamma) r}$. Let $\Gamma_{k}$ be the set of $\gamma \in \Gamma$ such that $k-1<d\left(x, \gamma^{-1} x\right) \leq k$. By the shadow lemma, there exist constants $d_{1}>0, C_{1}>0$ such that for all $\gamma \in \Gamma_{k}, e^{-\delta(\Gamma) k} \leq C_{1} \mu_{x_{0}}\left(O_{x_{0}}\left(\gamma^{-1} x_{0}, d_{1}\right)\right)$. On the other hand, there exists a constant $C_{2}>0([\mathrm{C}])$ such that for all $\zeta \in \partial H$, $\#\left\{\gamma \in \Gamma_{k} \mid \zeta \in O_{x_{0}}\left(\gamma^{-1} x_{0}, d_{1}\right)\right\} \leq C_{2}$. Therefore we have

$$
\sum_{\gamma \in \Gamma_{k}} \mu_{x_{0}}\left(O_{x_{0}}\left(\gamma^{-1} x_{0}, d_{1}\right)\right) \leq C_{2} \mu_{x_{0}}\left(\bigcup_{\gamma \in \Gamma_{k}} O_{x_{0}}\left(\gamma^{-1} x_{0}, d_{1}\right)\right) .
$$

Combining the above estimates we get $S_{k} \leq C_{1} C_{2} \mu_{x_{0}}(\partial H) e^{\delta(\Gamma) k}$, where $S_{k}=\# \Gamma_{k}$. Hence we have $N(k, x, x) \leq S_{1}+\cdots S_{k-1} \leq C e^{\delta(\Gamma) k}$ and the theorem is proved.

\section{Harmonic Densities}

(4.1) Preliminaries. A general reference for this section is [A1]. Consider a Hadamard manifold $H$ of dimension $n \geq 2$ with negative curvature $-\infty<-K_{2}^{2} \leq$ $K \leq-K_{1}^{2}<0$. Let

$$
\lambda_{1}=\inf \left\{\frac{\int_{M}|\nabla \varphi|^{2} d m}{\int \varphi^{2} d m}: \varphi \in C_{0}^{\infty}(H), \varphi \not \equiv 0\right\}
$$

be the first eigenvalue of the Laplacian operator $\triangle$. Then for each $\lambda<\lambda_{1}$ there exists a Green kernel $G_{\lambda}$ with respect to the operator $\triangle_{\lambda}=\triangle+\lambda I: G_{\lambda}: H \times H \rightarrow$ 
$(0, \infty]$. Set $G=G_{0}$; then we have

$$
\begin{array}{rlrl}
G(x, y) & \leq C e^{\beta d(x, y)} G_{\lambda}(x, y), & & \text { for } x, y \in H, 0<\lambda<\lambda_{1}, \\
G_{\lambda}(x, y) & \leq C e^{\beta^{\prime} d(x, y)}, & & \text { for } x, y \in H, d(x, y) \geq 1, \\
G_{\lambda}(x, y) \geq G(x, y)>C^{-1}, & & \text { for } x, y \in H, d(x, y) \leq 1, \quad \lambda>0,
\end{array}
$$

where $C, \beta, \beta^{\prime}$ are positive constants depending only on $n, K_{1}, K_{2}$ and $\lambda-\lambda_{1}$. More generally, for each $\lambda^{\prime}<\lambda<\lambda_{1}$ we have

$$
G_{\lambda^{\prime}}(x, y) \leq C e^{-\beta d(x, y)} G_{\lambda}(x, y), \quad \text { for } x, y \in H,
$$

where $C, \beta$ depend only on $K_{1}, K_{2}, n, \lambda, \lambda^{\prime}$.

Given any $0<\mu<\lambda_{1}$ and $\epsilon>0$, there exists $\alpha=\alpha(\epsilon, \mu, H)$ such that for all $0<\lambda<\lambda^{\prime}<\mu$ with $\left|\lambda-\lambda^{\prime}\right| \leq \alpha$,

$$
G_{\lambda}(x, y) \geq e^{-\epsilon d(x, y)} G_{\lambda^{\prime}}(x, y), \quad x, y, \in H, \quad d(x, y) \geq 1 .
$$

For each $\lambda<\lambda_{1}$ let $K_{\lambda}: H \times H \times \partial H \rightarrow R$ be the Poisson kernel of $\triangle_{\lambda}$. Then there is a bijection between the positive $\triangle_{\lambda}$-harmonic functions $u$ on $H$ and positive measures $\mu_{x}$ on $\partial H$ defined by: $u(y)=\int_{M} K_{\lambda}(x, y, \zeta) d \mu_{x}(\zeta)$. In general, we also have

$$
K_{\lambda}(x, y, \zeta)=\lim _{z \rightarrow \zeta} \frac{G_{\lambda}(y, z)}{G_{\lambda}(x, z)}
$$

Denote by $\nu_{x}$ the harmonic measure of $\triangle$. Then for $f \in L^{\infty}\left(\nu_{x}\right), 0<\lambda<$ $\lambda_{1}$, the map $u_{f, \lambda}(y)=\int_{\partial M} K_{\lambda}(x, y, \zeta) f(\zeta) d \nu_{x}(\zeta)$ defines a $\triangle_{\lambda}$-harmonic function satisfying $u_{f, \lambda}(y) \leq C\|f\|_{\infty} e^{-\alpha d(x, y)}$, where $C, \alpha$ are positive constants depending only on $n, K_{1}, K_{2}, \lambda$. Each positive $\triangle_{\lambda}$-harmonic function $u$ is a $\triangle_{\lambda}$-potential, i.e., $u(y)=\lambda \int_{H} G(x, y) u(x) d x$. If $\varphi_{\lambda}(y) \triangleq \int_{\partial H} K_{\lambda}(x, y, \zeta) d \nu_{x}(\zeta)$ then for $\nu_{x}$-a.e. $\zeta \in$ $\partial H, \frac{u(x)}{\varphi_{\lambda}(x)}$ is bounded on the geodesic ray $\overrightarrow{O \zeta}$. If $u$ is a $\triangle_{\lambda^{\prime}}$-harmonic positive function and $\lambda^{\prime}<\lambda$, then $\frac{u(y)}{\phi_{\lambda}(y)} \rightarrow 0$ as $y \rightarrow \zeta$ radially for $\nu_{x}$-a.e. $\zeta \in \partial H$.

(4.2) Construction of a harmonic density. Consider a Fuchsian group $\Gamma$ and $M=H / \Gamma$. Denote by $\lambda(\Gamma)$ the first eigenvalue of $\triangle$ on $M$; then $\lambda(\Gamma) \leq \lambda_{1}$. We assume that $\lambda(\Gamma)<\lambda_{1}$. Then the Green series $\sum_{\gamma \in \Gamma} G_{\lambda}(\gamma x, y)$ diverges for all $\lambda(\Gamma)<\lambda<\lambda_{1}$ and converges for all $\lambda<\lambda(\Gamma)$. According to (4.1) (5), the series $\sum_{\gamma \in \Gamma} e^{\epsilon d(x, \gamma x)} G_{\lambda(\Gamma)}(\gamma x, y)$ is divergent for all $\epsilon>0$ and $y \in H \backslash \Gamma(x)$. Fix an increasing sequence $R_{i} \rightarrow \infty$ and a decreasing sequence $\epsilon_{i} \rightarrow 0$ and consider the following function $h: R^{+} \rightarrow R^{+}: h\left(R_{1}\right)=0, \quad h(t)=\epsilon_{i}\left(t-R_{i}\right)+h\left(R_{i}\right)$ for $R_{i} \leq t<R_{i+1}$. If $R_{i}$ is large enough, then we have

$$
\sum_{\substack{\gamma \in \Gamma \\ R_{i} \leq d(\gamma x, x)<R_{i+1}}} e^{h(d(x, \gamma x))} G_{\lambda(\Gamma)}(\gamma x, y) \geq 1 .
$$

Now fix $y_{0} \in H \backslash \Gamma(x)$ and consider the following series of measures:

$$
\nu_{y}^{\lambda} \triangleq \frac{\sum_{\gamma \in \Gamma} e^{h(d(x, \gamma x))} G_{\lambda}(\gamma x, y) \delta_{\gamma x}}{\sum_{\gamma \in \Gamma} e^{h(d(x, \gamma x))} G_{\lambda}\left(\gamma x, y_{0}\right)},
$$


where $\delta_{\gamma x}$ is the Dirac measure at $\gamma x$. Take a weak limit $\lim _{\lambda_{i} \rightarrow \lambda(\Gamma)-} \nu_{y_{0}}^{\lambda_{i}}=\nu_{y_{0}}$ (since $\nu_{y_{0}}^{\lambda}$ are probability measures weak limits exist). Then it is easy to see that for any $y \in H \backslash \Gamma(x), \lim _{\lambda_{i} \rightarrow \lambda(\Gamma)-} \nu_{y}^{\lambda_{i}}$ also exists and we denote it by $\nu_{y}$. From the construction and the relation (4.1) (6) we have:

(i) $\nu_{y}$ is supported on $L(\Gamma)$.

(ii) $\frac{d \nu_{y}}{d \nu_{y_{0}}}(\zeta)=K_{\lambda(\Gamma)}\left(y_{0}, y, \zeta\right)$ for all $y \in H, \zeta \in L(\Gamma)$.

Theorem (4.2.1) (see Ancona [A2]). (i) There exists a $\lambda(\Gamma)$-harmonic density $\nu$ of $\Gamma$, where $\lambda(\Gamma)$ is the first eigenvalue of Laplacian on $M=H / \Gamma$. The following defined function is a $\lambda(\Gamma)$-harmonic function on $M$ :

$$
\varphi_{\nu}(y)=\int K_{\lambda(\Gamma)}\left(y_{0}, y, \zeta\right) d \nu_{y_{0}}(\zeta)
$$

(ii) If $\Gamma$ has a fundamental domain $D$ in $H$ with $\bar{D} \cap L(\Gamma)=\varnothing$, then the series $\sum_{\gamma \in \Gamma} G_{\lambda(\Gamma)}(\gamma x, y)$ diverges for all $y \in H$, and $\varphi_{\nu}$ is the unique $\lambda(\Gamma)$-harmonic function on $M$ satisfying $\varphi_{\nu}\left(y_{0}\right)=1$. If moreover, $L(\Gamma) \neq S_{\infty}$, then $\lambda(\Gamma)>0$.

The proof of the following statement is the same as in section (3.3).

Proposition (4.2.2). Let $\sigma$ be a $\lambda$-harmonic density of $\Gamma$. Then $\Gamma$ is ergodic with respect to the measure class defined by $\sigma$ if and only if any other $\lambda$-harmonic density coincides with $\sigma$ up to a scalar multiplication.

(4.3) A version of the Ahlfors theorem for harmonic densities. Let $\left\{\nu_{x}\right\}_{x \in H}$ be the family of harmonic measures of the Laplacian $\triangle$. Let $\Gamma$ be a Fuchsian group. Next we prove a theorem similar to that of Ahlfors for Kleinian groups $([\mathrm{Ah}])$. Compare also with Theorem (3.5.5).

Theorem (4.3.1). The harmonic measure $\nu_{x}\left(L^{r}(\Gamma)\right)$ of the radial limit set of any Fuchsian group is either 1 or 0.

Proof. Let $\chi_{\Gamma}^{r}$ be the characteristic function of the radial limit set $L^{r}(\Gamma)$. Then

$$
\varphi(x) \triangleq \int_{\partial M} \chi_{\Gamma}^{r}(\zeta) d \nu_{x}(\zeta)=\nu_{x}\left(L^{r}(\Gamma)\right)
$$

defines a $\Gamma$-invariant harmonic function with $0 \leq \varphi(x) \leq 1$.

If $\nu_{x}\left(L^{r}(\Gamma)\right)$ is positive, then by Fatou's radial convergence theorem, there exists at least one $\zeta \in L^{r}(\Gamma)$ such that $\lim _{t \rightarrow \infty} \varphi(v(t))=\chi_{\Gamma}^{r}(\zeta)=1$, where $v(t)$ is a geodesic ray in $H$ so that $v(\infty)=\zeta$. Since $\zeta \in L^{r}(\Gamma)$, there exist sequences $\left\{\gamma_{n}\right\} \subset \Gamma$ and $t_{n} \rightarrow \infty$ such that $d\left(\gamma_{n} v\left(t_{n}\right), v(0)\right) \leq C$ where $C$ is a positive constant. By compactness we can assume moreover that $\gamma_{n} v\left(t_{n}\right) \rightarrow x_{0} \in H$. The $\Gamma$-invariance and continuity of $\varphi$ imply that $\varphi\left(x_{0}\right)=1$. Hence $\varphi$ attains a maximum at an interior point $x_{0}$ and it has to be the constant function 1 .

The following theorem follows from the same idea because the Fatou's radial convergence theorem is true for any $\lambda$-harmonic function.

Theorem (4.3.2). Let $\sigma$ be any $\lambda$-harmonic density for a Fuchsian group $\Gamma, \lambda<$ $\lambda_{1}$. Then either $\sigma_{x}\left(L^{r}(\Gamma)\right)=1$ or $\sigma_{x}\left(L^{r}(\Gamma)\right)=0$. 
Proof. (Compare our proof with [S1], see also [A2] for more details about the Fatou theorem.) Let $\Phi(x)$ be the total mass of $\sigma_{x}$. Then $\Phi$ is an eigenfunction of the Laplacian on $H$ with eigenvalue $\lambda$. Consider the $\Phi$-process corresponding to the operator $L=\triangle+2 \operatorname{grad} \log \Phi$. The transition probability for this process is $P_{t}^{\Phi}(x, y)=e^{\lambda t} \frac{P_{t}(x, y)}{\Phi(x) \Phi(y)}$ (where $P_{t}(x, y)$ is the heat kernel of $\triangle$ on $H$ ) and the $\Phi$-process preserves the measure $\Phi^{2}(y) d y$ on $H$. It is easy to check that the positive $L$-harmonic functions are just the functions $\frac{g}{\Phi}$, where $g$ is a positive eigenfunction of $L$ with eigenvalue $\lambda$, and the $L$-harmonic measures (i.e. the hitting probability at $\partial H$ of the $\Phi$-biased random motion starting at $x \in H$ ) are exactly the measures $\frac{\sigma_{x}}{\Phi(x)}$. By the Fatou theorem, the radial limit of any positive $L$-harmonic function exists $\sigma_{x}$-almost everywhere. The remaining part of the proof is the same as in Theorem (4.3.1).

Remark. The condition $\lambda<\lambda_{1}$ implies that the operator $L=\varphi^{-1}(\triangle+\lambda I) \varphi$ is Greenian. Consequently we can use the Fatou theorem in the above argument. (See [A2] for the properties of a Greenian operator.)

\section{The ACtion of $\Gamma$ on $\partial H \times \partial H$ AND THE GEODESIC FLOW}

(5.1) The topological picture. In this section we continue to assume that $\Gamma$ is a Fuchsian group. $\Gamma$ has a natural diagonal action on $\partial H \times \partial H: \gamma(\zeta, \eta)=(\gamma \zeta, \gamma \eta)$. This action is closely related with the geodesic flow $g^{t}$ on $S M$, the unit tangent bundle of $M=H / \Gamma$. Recall that a point $v \in S M$ is called non-wandering if for any neighborhood $O$ of $v$ we can find a sequence $t_{n} \rightarrow \infty$, such that $g^{t_{n}} O \cap O \neq \varnothing$. From this definition it is easy to see that $v$ is non-wandering if and only if both $v(-\infty)$ and $v(+\infty)$ are in $L(\Gamma)$. From now on we denote by $\Omega$ the set of non-wandering points in $S M$. There is a natural identification $\Omega=[(L(\Gamma) \times L(\Gamma) \backslash$ diagonal $) \times R] / \Gamma$. We first recall the following two results by Eberlein ([E1]).

Proposition (5.1.1). The action of $\Gamma$ on $L(\Gamma) \times L(\Gamma)$ is topologically transitive; i.e., there exists a dense orbit.

Proposition (5.1.2). Let $M=H / \Gamma$ be a Fuchsian manifold. Then we have

(1) $g^{t}$ is topologically transitive on $\Omega$.

(2) Periodic vectors are dense in $\Omega$.

(3) Either $\Omega=S M$ or $\Omega$ is connected and nowhere dense in $S M$.

These results provides a clear picture of the topological dynamics of the geodesic flow and the $\Gamma$ action on its limit set. But the corresponding ergodic theory for the geodesic flow and the $\Gamma$ action turns out to be much more complicated. Our main purpose in this section is to construct geometrically meaningful invariant measures of the geodesic flow and to study their ergodicity.

(5.2) Invariant measures. Unlike the case of the $\Gamma$ acting on $\partial H$, there are plenty of invariant measures of the diagonal $\Gamma$ action on $\partial H \times \partial H$. Obviously, there is a natural one-one correspondence between the following two sets of invariant measures:

$\left\{\right.$ All $g^{t}$-invariant measures on $\left.S M\right\} \stackrel{1-1}{\leftrightarrow}\{$ All $\Gamma$-invariant measures on $\partial H \times \partial H\}$.

Following [K], [L1], we construct two classes of $\Gamma$-invariant measures on $\partial H \times \partial H$. 
Proposition (5.2.1). Let $\sigma$ be any $D$-conformal density of $\Gamma$. Then the measure $d U_{x}^{\sigma}$ on $\partial H \times \partial H$ defined by $d U_{x}^{\sigma}(\zeta, \eta)=e^{D \beta_{x}(\zeta, \eta)} d \sigma_{x}(\zeta) d \sigma_{x}(\eta)$, where $\beta_{x}(\zeta, \eta)=$ $\rho_{x, \zeta}(y)+\rho_{x, \eta}(y)$ for any point $y$ on the geodesic from $\zeta$ to $\eta$ is a locally finite invariant measure of the diagonal action of $\Gamma$.

Proof. By definition, we have

$$
\begin{gathered}
\frac{d U_{x}^{\sigma}(\gamma \zeta, \gamma \eta)}{d\left(\gamma^{*} U_{x}^{\sigma}\right)(\gamma \zeta, \gamma \eta)}=\frac{e^{D \beta_{x}(\gamma \zeta, \gamma \eta)} d \sigma_{x}(\gamma \zeta) d \sigma_{x}(\gamma \eta)}{e^{D \beta_{x}(\zeta, \eta)} d\left(\gamma^{*} \sigma_{x}\right)(\gamma \zeta) d\left(\gamma^{*} \sigma_{x}\right)(\gamma \eta)} \\
\quad=\frac{e^{D \beta_{x}(\gamma \zeta, \gamma \eta)}}{e^{D \beta_{x}(\zeta, \eta)}} e^{D\left(\rho_{\left.\gamma^{-1} x_{x, \zeta}(x)+\rho_{\gamma^{-1} x, \eta}(x)\right)}\right.}=1 . \quad \square
\end{gathered}
$$

Remark. Obviously we have $d U_{x}^{\sigma}=d U_{y}^{\sigma}$ for any $x, y \in H$. In other words, the measure does not depend on the choice of base points. From now on we will denote it by $d U^{\sigma}$. We denote the corresponding invariant measure of the geodesic flow on $S M$ by $d \mu^{\sigma}$.

Fix a point $o \in H$. Let $\sigma_{o}$ be an $D$-conformal density of $\Gamma$. For each $v \in S H$, the following map $P: W^{s u}(v) \longrightarrow \partial H-v(-\infty), P(w)=w(\infty)$, is a homeomorphism. One can first pull the measure $\sigma_{o}$ back to $W^{s u}(v)$ by the map $P$ and then define a measure $\mu^{s u}$ on $W^{s u}(v)$ by

$$
\frac{d \mu^{s u}}{d\left(P^{*} \sigma_{o}\right)}(w)=e^{-D \rho_{o, w(\infty)}(w(0))} .
$$

It is easy to check that the above equation is well-defined and is $\Gamma$-equivariant. Thus it can be projected to the quotient unit tangent bundle $S M$ to yield a family of measures on each $W^{s u}(v)$ satisfying

$$
g_{t}^{*} \mu^{s u}=e^{D t} \mu^{s u} .
$$

One then obtains a family of measures $\mu^{s s}$ on leaves of the $W^{s s}(v)$ foliation with the property that $g_{t}^{*} \mu^{s s}=e^{-D t} \mu^{s s}$ via pulling over by the flip map $v \mapsto-v$. Define the measures $\mu^{u}, \mu^{s}$ by

$$
d \mu^{u}=d \mu^{s u} \times d t, d \mu^{s}=d \mu^{s s} \times d t .
$$

It is easy to see that $\mu^{u}$ and $\mu^{s}$ are holonomy invariant and moreover

$$
d \mu^{\sigma}=d \mu^{u} \times d \mu^{s s}=d \mu^{s} \times d \mu^{s u} .
$$

Due to the similarity between the above construction and the Margulis construction in [M], we call $\mu^{\sigma}$ a Bowen-Margulis measure for the geodesic flow on $S M$.

On the other hand, given any invariant measure $\mu$ of the geodesic flow on $S M$ satisfying $(5.2 .1 *)(5.2 .2 *)(5.2 .3 *)$, one can construct a $D$-conformal density $\sigma$ of $\Gamma$, such that $\mu=\mu^{\sigma}$ (for the construction when $\Gamma$ is a cocompact lattice, see [L2]).

Next we consider the corresponding construction for harmonic measures.

Proposition (5.2.2). Let $\sigma$ be any $\lambda$-harmonic density of $\Gamma$. Then the measure $d \Lambda_{x}^{\sigma}(\zeta, \eta)=G_{x}(\zeta, \eta) d \sigma_{x}(\zeta) d \sigma_{x}(\eta)$, where

$$
G_{x}(\zeta, \eta)=\lim _{\substack{y_{1} \rightarrow \zeta \\ y_{2} \rightarrow \eta}} \frac{G_{\lambda}\left(y_{1}, y_{2}\right)}{G_{\lambda}\left(x, y_{1}\right) G_{\lambda}\left(x, y_{2}\right)}
$$

is a $\Gamma$-invariant measure on $\partial H \times \partial H$.

Proof. By definition, we have $\frac{d \Lambda_{x}^{\sigma}}{d\left(\gamma^{*} \Lambda_{x}^{\sigma}\right)}(\gamma \zeta, \gamma \eta)=\frac{G_{x}(\gamma \zeta, \gamma \eta) d \sigma_{x}(\gamma \zeta) d \sigma_{x}(\gamma \eta)}{G_{x}(\zeta, \eta) d\left(\gamma^{*} \sigma_{x}\right)(\gamma \zeta) d\left(\gamma^{*} \sigma_{x}\right)(\gamma \eta)}=$ $\frac{G_{x}(\gamma \zeta, \gamma \eta)}{G_{x}(\zeta, \eta)} K_{\lambda}\left(\gamma^{-1} x, x, \zeta\right) K_{\lambda}\left(\gamma^{-1} x, x, \eta\right)=1$ (compare (4.1)). 
Note that the measure $\Lambda_{x}^{\sigma}$ also does not depend on the choice of $x$. We denote it by $\Lambda^{\sigma}$. We denote the corresponding invariant measure of the geodesic flow on $S M$ by $\nu^{\sigma}$.

(5.3) Ergodic theory of the infinite Bowen-Margulis measure. Consider a separable, complete metric space $(X, d)$ and a $\sigma$-finite Borel measure $\mu$ on $X$ which is invariant under a continuous flow $T_{s}: X \rightarrow X$. The space $X$ is not necessarily compact and the measure $\mu$ might be infinite. However, we assume that $X$ is locally compact and that the $\mu$ measure of each compact subset of $X$ is finite.

We recall the following Hopf generalization of the Birkhoff ergodic theorem.

Theorem (5.3.1). If $f, g \in L^{1}(\mu), g>0$ and $\lim _{T \rightarrow 0} \int_{0}^{T} g\left(T_{s} x\right) d s=+\infty$ for $\mu$-a.e. $x$ in $X$ (such a function $g$ does not always exist), then the limit

$$
h(x)=\lim _{T \rightarrow \infty} \frac{\int_{0}^{T} f\left(T_{s} x\right) d s}{\int_{0}^{T} g\left(T_{s} x\right) d s}
$$

exists $\mu$-almost everywhere and moreover, $h$ is measurable and flow invariant, $g h \in L^{1}(\mu)$ and for any bounded, measurable $T_{s}$-invariant function $v$, we have $\int_{X} g h v d \mu=\int_{X} f v d \mu$.

The measure $\mu$ is said to be $T_{s}$ ergodic if for any $T_{s}$ invariant measurable set $A \subset X$, either $\mu(A)=0$ or $\mu(X \backslash A)=0$.

Proposition (5.3.2). If $\mu$ is $T_{s}$ ergodic under the hypotheses of Proposition (3.3.1), then for almost all $x \in X$, we have

$$
\lim _{T \rightarrow \infty} \frac{\int_{0}^{T} f\left(T_{s} x\right) d s}{\int_{0}^{T} g\left(T_{s} x\right) d s}=\frac{\int_{X} f d \mu}{\int_{X} g d \mu} .
$$

Corollary (5.3.3). If $\mu$ is ergodic and $A, B$ are two measurable subsets of $X$, $\mu(A)<\infty, \mu(B) \neq 0$ then we have

$$
\lim _{T \rightarrow \infty} \frac{\int_{0}^{T} \chi_{A}\left(T_{t} x\right) d t}{\int_{0}^{T} \chi_{B}\left(T_{t} x\right) d t}=\frac{\mu(A)}{\mu(B)} .
$$

An important notion for a dynamical system on a noncompact space is the notion of conservative set.

Definition (5.3.4). A point $x \in X$ is called a dissipative point for $T_{s}$ if for any compact set $A \subset X$, there exists a $t_{0}>0$ such that $T_{t} x \notin A$ for all $t \geq t_{0}$. Otherwise $x$ is called conservative. We denote by $C^{T_{s}}$ and $D^{T_{s}}$ the set of all conservative and dissipative points in $X$. The flow $T_{s}$ is said to be conservative with respect to $\mu$ if $\mu\left(D^{T_{s}}\right)=0$.

Proposition (5.3.5). The conservative set of the geodesic flow $g^{t}$ on $S M$ is exactly the $\Gamma$-quotient of $\left(\partial H \times L^{r}(\Gamma) \backslash\right.$ diagonal $) \times R$, i.e., the set of vectors $v$ whose end asymptotic point $v(+\infty)$ is a radial limit point of $\Gamma$.

Proof. If $v \in C^{g_{t}}$, then there exists compact set $A \subset S M$ so that $g^{t_{i}} v \in A$ for a sequence $t_{i} \rightarrow \infty$. It follows that there exist $\gamma_{i} \in \Gamma$ such that the geodesic $v(t)$ in the universal covering satisfies $\dot{v}\left(t_{i}\right) \in \gamma_{i} A$. Hence $v(\infty)$ is a radial limit point. On the other hand, if $v(\infty) \in L^{r}(\Gamma)$, then $v(t)$ lies in a $K$-neighborhood of $\Gamma x$ and thus on $S M, g^{t} v$ returns infinitely often to the $K$-neighborhood of $S_{x} M$. 
Proposition (5.3.6). If $\sigma$ is a D-conformal density of $\Gamma$ with $\sigma\left(L^{r}(\Gamma)\right)>0$, then $g^{t}$ is conservature with respect to the invariant measure $d \mu^{\sigma}=d U^{\sigma} d t$.

Proof. By Corollary (3.5.6), $\sigma\left(L^{r}(\Gamma)\right)=\sigma(L(\Gamma))$. Therefore $d \mu^{\sigma}$ has full measure on the conservative set.

Theorem (5.3.7). Let $\sigma$ be a $D$-conformal density of $\Gamma$. Then we have

(i) $g^{t}$ is either completely dissipative or completely conservative with respect to $d \mu^{\sigma}$.

(ii) $g^{t}$ is conservative with respect to $d \mu^{\sigma}$ if and only if $\sum_{\gamma \in \Gamma} e^{-D d(x, \gamma x)}=+\infty$.

Proof. (i) If $d \mu^{\sigma}$ gives positive measure to the conservative set, then $\sigma_{x}\left(L^{r}(\Gamma)\right)>0$ and by (3.5.6) it has full measure $\Rightarrow g^{t}$ is completely conservative.

(ii) If $\sum_{\gamma \in \Gamma} e^{-D d(x, \gamma x)}<\infty$, then by Proposition (3.5.1), $\sigma_{x}\left(L^{r}(\Gamma)\right)=0$ and consequently $g^{t}$ is completely dissipative.

The most difficult part is to show that divergence of the Poincaré series implies that the geodesic flow is conservative. The idea of our proof is due to Sullivan ([S2]). See also $[\mathrm{N}]$ for a nice treatment of the constant curvature case. Fix a point $x \in H$. Let $t_{0}$ be the largest point mass of $\sigma_{x}$. By the proof of (3.4.1), there exists $R>0$ such that for all $y \in H$ with $d(y, x)>2 R$,

$$
\sigma_{x}\left(\triangle_{1}(y)\right) \geq \frac{1}{2}\left(\sigma_{x}(\partial H)-t_{0}\right) \triangleq C_{1}>0,
$$

where $\triangle_{1}(y)=\{\zeta \in \partial H \mid \zeta=v(\infty), v(0) \in B(y, R), v(t) \in B(x, R)$ for some $t>$ $0\}$ (this is because, if $R$ is large enough, then the $d_{x}^{\epsilon}$ diameter of $\partial H \backslash \triangle_{1}(y)$ is small). Since there are only finitely many $\gamma \in \Gamma$ such that $\gamma(B(x, R)) \cap B(x, R) \neq \varnothing$, we may assume that $\gamma(B(x, R)) \cap B(x, R)=\varnothing$ for all $i d \neq \gamma \in \Gamma$ without affecting the divergence of $\sum_{\gamma \in \Gamma} e^{-D d(x, \gamma x)}$. Denote $\triangle_{2}(\gamma x)=\{\zeta \in \partial H \mid \zeta=$ $v(\infty)$ for some vector $v$ such that $v(0) \in B(x, R), v(t) \in B(\gamma x, R)$ for some $t>0\}$. Then we have $\sigma_{x}\left(\gamma^{-1}\left(\triangle_{2}(\gamma x)\right)\right) \geq C_{1}$ by (1) and consequently, $\sigma_{x}\left(\triangle_{2}(\gamma x)\right)$ $=\sigma_{\gamma^{-1} x}\left(\gamma^{-1}\left(\triangle_{2}(\gamma x)\right)\right)=\int_{\gamma^{-1} \triangle_{2}(\gamma x)} e^{-D \rho_{x, \zeta}\left(\gamma^{-1} x\right)} d \sigma_{x}(\zeta)$. Since $\rho_{x, \zeta}\left(\gamma^{-1} x\right) \leq$ $d\left(\gamma^{-1} x, x\right)$ and moreover, since there exists a universal constant $B_{1}$ such that $\rho_{x, \zeta}\left(\gamma^{-1} x\right) \geq d\left(x, \gamma^{-1} x\right)-B_{1}$ for all $\zeta \in \gamma^{-1} \triangle_{2}(\gamma x)$, we can find a constant $A_{1}>0$ such that

$$
A_{1} e^{-D d\left(x, \gamma^{-1} x\right)} \geq \sigma_{x}\left(\triangle_{2}(\gamma x)\right) \geq C_{1} e^{-D d\left(x, \gamma^{-1} x\right)} .
$$

Denote by $\tilde{O}$ the unit tangent bundle of $B(x, R)$ in $S H$ and by $O$ its projection to $S M$. Then by (1) (2) and the definition of $\mu^{\sigma}$ it is easy to see that $A_{2} e^{-D d\left(x, \gamma^{-1} x\right)} \geq$ $\mu^{\sigma}\left[\tilde{O} \cap g^{-d(x, \gamma x)} \gamma(\tilde{O})\right] \geq C_{2} e^{-D d\left(x, \gamma^{-1} x\right)}$ for all $\gamma \in \Gamma$, where $C_{2}=C_{2}(H, R, \Gamma)$ and $A_{2}=A_{2}(H, R, \Gamma)$ are constants. If we assume that $R>1$ and $k \leq d(x, \gamma x)<k+1$ then we have

$$
C_{3} e^{-D d\left(x, \gamma^{-1} x\right)} \geq \mu^{\sigma}\left[\tilde{O} \cap g^{-k} \gamma(\tilde{O})\right] \geq C_{3}^{-1} e^{-D d\left(x, \gamma^{-1} x\right)},
$$

for some constant $C_{3}=C_{3}(H, R, \Gamma)$. From the divergence of the Poincaré series we get $\sum_{n=1}^{\infty} \mu^{\sigma}\left(\tilde{O} \cap g^{-n}(\Gamma(\tilde{O}))\right)=\infty$. Now let $E_{n}$ be the event of $O \cap g^{-n}(O)$ (i.e., 
the event that a point of $O$ returns to $O$ at time $n$ by the geodesic flow). Then its probability is given by

$$
P\left(E_{n}\right)=\frac{\mu^{\sigma}\left(O \cap g^{-n}(O)\right)}{\mu^{\sigma}(O)}=\frac{\mu^{\sigma}\left(\tilde{O} \cap g^{-n}(\Gamma \tilde{O})\right)}{\mu^{\sigma}(\tilde{O})} .
$$

Notice that $P\left(E_{n+m} \cap E_{n}\right)=P\left(E_{n}\right) P\left(E_{n+m} \mid E_{n}\right)$. Moreover, using the elementary inequality $\frac{a_{1}+a_{2}+\cdots+a_{k}}{b_{1}+b_{2}+\cdots+b_{k}} \leq \max _{1 \leq j \leq k}\left\{\frac{a_{j}}{b_{j}}\right\}$, we can find a $\gamma \in \Gamma$, such that

$$
\begin{aligned}
P\left(E_{n+m} \mid E_{n}\right) & =\frac{\mu^{\sigma}\left(\Gamma(\tilde{O}) \cap g^{n}(\tilde{O}) \cap g^{-m} \Gamma(\tilde{O})\right)}{\mu^{\sigma}\left(\Gamma(\tilde{O}) \cap g^{n}(\tilde{O})\right)} \\
& \leq \frac{\mu^{\sigma}\left(\gamma(\tilde{O}) \cap g^{n}(\tilde{O}) \cap g^{-m} \Gamma(\tilde{O})\right)}{\mu^{\sigma}\left(\gamma(\tilde{O}) \cap g^{n}(\tilde{O})\right)} \\
& =\frac{\mu^{\sigma}\left(\tilde{O} \cap g^{n}\left(\gamma^{-1} \tilde{O}\right) \cap g^{-m}(\Gamma \tilde{O})\right)}{\mu^{\sigma}\left(\tilde{O} \cap g^{n}\left(\gamma^{-1} \tilde{O}\right)\right)} .
\end{aligned}
$$

Let $\Gamma_{1}=\left\{\beta \in \Gamma \mid \tilde{O} \cap g^{n}\left(\gamma^{-1} \tilde{O}\right) \cap g^{-m}(\beta \tilde{O}) \neq \varnothing\right\}$. Then by the above argument there exists a constant $C_{4}, C_{5}$ such that for each $\beta \in \Gamma_{1}$, we have

i) $\mu^{\sigma}\left(\tilde{O} \cap g^{n}\left(\gamma^{-1} \tilde{O}\right) \cap g^{-m}(\beta \tilde{O})\right) \leq \mu^{\sigma}\left(g^{n} \gamma^{-1} \tilde{O} \cap g^{-m}(\beta \tilde{O})\right) \leq C_{4} e^{-D(n+d(x, \beta x))}$.

ii) $\mu^{\sigma}\left(\tilde{O} \cap g^{n}\left(\gamma^{-1} \tilde{O}\right)\right) \geq C_{4}^{-1} e^{-n D}$.

Consequently we get

$$
\begin{aligned}
\mu^{\sigma}\left(\tilde{O} \cap g^{n}\left(\gamma^{-1} \tilde{O}\right) \cap g^{-m}(\Gamma \tilde{O})\right) & \leq \sum_{\beta \in \Gamma_{1}} C_{4} e^{-D(n+d(x, \beta x))} \\
& \leq C_{4} e^{-n D} \sum_{\beta \in \Gamma_{1}} e^{-D d(x ; \beta x)} \\
& \leq C_{4} C_{5} e^{-n D} \sum_{\beta \in \Gamma_{1}} \mu^{\sigma}\left(\tilde{O} \cap g^{-m}(\beta \tilde{O})\right) \\
& \leq C_{6} e^{-n D} P\left(E_{m}\right),
\end{aligned}
$$

for some constant $C_{6}$. Therefore we have

$$
P\left(E_{n+m} \mid E_{n}\right) \leq \frac{C_{6} e^{-n D} P\left(E_{m}\right)}{C_{4}^{-1} e^{-n D}}=C_{4} C_{6} P\left(E_{m}\right) .
$$

Thus $P\left(E_{n+m} \cap E_{n}\right) \leq C P\left(E_{n}\right) P\left(E_{m}\right)$ for some constant $C>0$. By the BorelCantelli lemma (see [S4] for a generalized version) we have $P\left(E_{n}\right.$ infinitely often $)>$ 0. Combining this and Corollary (3.5.6) we have that almost every $x \in O$ is a conservative point for $g^{t}$. Hence the theorem is proved.

Corollary (5.3.8). Let $\sigma$ be a D-conformal density of $\Gamma$. Then $\sigma_{x}\left(L^{r}(\Gamma)\right)=$ $\sigma_{x}(L(\Gamma))$ if and only if $\sum_{\gamma \in \Gamma} e^{-D d(x, \gamma x)}=\infty$.

We now begin to study the ergodicity problem. Consider the function $\tau: S M \rightarrow$ $R^{+}, \tau(v)=d(v(0), x)$, where $d$ is the Riemannian metric on $M$. Let $O_{r}=\tau^{-1}[0, r]$. We want to show that

$$
\varlimsup_{r \rightarrow \infty} \frac{1}{r} \log \mu^{\sigma}\left(O_{r}\right)<\infty .
$$

If $\ell$ is a geodesic passing through $B(x, r)$, then the length of the intersection $\ell \cap B(x, r)$ is $\leq 2 r$ and we also have $d(x, \ell) \leq r$. By Proposition (1.2.1), there exists 
a constant $C_{1}=C_{1}(H)$ such that $\beta_{x}(\ell(-\infty), \ell(\infty)) \leq C_{1} e^{2 r}$. Since $d \mu^{\sigma}(\zeta, \eta)=$ $e^{D \beta_{x}(\zeta, \eta)} d \sigma_{x}(\zeta) d \sigma_{x}(\eta) d t$ we have $\mu^{\sigma}\left(B_{r}\right) \leq C_{2} r e^{2 D r}$, where $C_{2}=C_{2}(H)$ is a constant and $B_{r}$ denotes the unit tangent bundle of $B(x, r)$. But the ball $B_{r}$ covers $O_{r}$; therefore $(*)$ is proved. Now consider the function $\rho=e^{-(2 D+\epsilon) \tau}$ for some $\epsilon>0$. We have the following estimates:

$$
\left|\frac{d \rho}{\rho}\right| \leq(2 D+\epsilon)|d \tau| \leq 2 D+\epsilon .
$$

By $(*), \rho \in L^{1}\left(\mu^{\sigma}\right)$. Hence we have:

Lemma (5.3.9). There exists a positive $\mu^{\sigma}$-integrable function $\rho$ on $S M$ which satisfies, for some constant $C$,

$$
\frac{\rho(v)-\rho(w)}{\rho(w)} \leq C d(v(0), w(0)) .
$$

This lemma allows us to prove an analogy of Hopf's ergodic theorem for geodesic flows.

Theorem (5.3.10). If the geodesic flow is conservative with respect to $\mu^{\sigma}$ then it is ergodic with respect to the same measure.

Proof. The idea of our proof is due to Hopf. By the conservative condition we have $\int_{0}^{\infty} \rho\left(g^{t} v\right) d t=+\infty$ for $\mu^{\sigma}$-almost all $v \in S M$. By Theorem (5.3.1), the following limit,

$$
\lim _{T \rightarrow \infty} \frac{\int_{0}^{T} f\left(g^{t} v\right) d t}{\int_{0}^{T} \rho\left(g^{t} v\right) d t}=f_{\rho}(v),
$$

exists for each $f \in L^{1}\left(d \mu^{\sigma}\right)$ and $\mu^{\sigma}$-almost all $v \in S M$. The measure $\mu^{\sigma}$ is ergodic if and only if $f_{\rho}$ is constant almost everywhere for all $f \in L^{1}\left(d \mu^{\sigma}\right)$. It suffices to consider only the set of continuous functions $f$ with compact support (they are dense in $\left.L^{1}\left(d \mu^{\sigma}\right)\right)$. For continuous functions it is easy to see that $f_{\rho}(v)=f_{\rho}(w)$ if $v \in W^{s}(w)$ or $v \in W^{u}(w)$. Hence $f_{\rho}$ determines a $\Gamma$-invariant function on $\partial H \times \partial H \backslash$ diagonal which is almost everywhere constant on each factor $\zeta \times \partial H$ or $\partial H \times \eta$. Therefore it is constant $d U^{\sigma}$-almost everywhere by the Fubini theorem and the loca product structure of the measure $d U^{\sigma}$.

Corollary (5.3.11). If $\mu^{\sigma}(S M)<\infty$, then we have:

(1) $\sigma$ is the unique $\Gamma$-conformal density of dimension $\delta(\Gamma)$.

(2) $\sigma_{x}\left(L^{r}(\Gamma)\right)=\sigma_{x}(L(\Gamma))$.

(3) $g^{t}$ is ergodic with respect to $\mu^{\sigma}$.

Proof. Since $\mu^{\sigma}(S M)<\infty$, the Poincaré recurrence theorem implies that $\mu^{\sigma}$ almost all points $v \in S M$ are conservative. Thus $\sigma_{x}\left(L^{r}(\Gamma)\right)>0$ and the corollary follows from Corollary (3.5.6) and Theorem (5.3.10).

(5.4) A finiteness criterion for the Bowen-Margulis measure. By Corollary (5.3.11), if the Bowen-Margulis measure $\mu^{\sigma}$ for a Fuchsian group has finite total mass, then it is ergodic and the radial limit set has full measure. The purpose of this section is to present a finiteness criterion, which, in the constant curvature case, is due to Sullivan ([S1]). First we prove the following simple lemma. 
Lemma (5.4.1). Let $\Gamma$ be a Fuchsian group and $B$ a bounded subset of $S M$. Then there exists a constant $C$ such that for $\mu^{\sigma}$-almost all $v \in S M$,

$$
\lim _{T \rightarrow \infty} \frac{1}{T} \int_{0}^{T} \chi_{B}\left(g^{t} v\right) d t=C .
$$

Moreover, $C=0$ unless $\mu^{\sigma}(S M)<\infty$.

Proof. If $g^{t}$ is dissipative then almost all trajectories spend only a finite amount of time in $B$; therefore we have $\lim _{T \rightarrow \infty} \frac{1}{T} \int_{0}^{T} \chi_{B}\left(g^{t} v\right) d t=0$. If $g^{t}$ is conservative then it is ergodic and by the ergodic theorem, the following limit,

$$
\lim _{T \rightarrow \infty} \frac{1}{T} \int_{0}^{T} \chi_{B}\left(g^{t} v\right) d t=\frac{\mu^{\sigma}(B)}{\mu^{\sigma}(S M)},
$$

exists and is equal to 0 unless $\mu^{\sigma}(S M)<\infty$.

Theorem (5.4.2). Let $\sigma$ be an $\alpha$-conformal density of $\Gamma$. If $\mu^{\sigma}(S M)=\infty$, then $\lim _{T \rightarrow \infty} \frac{1}{T} \sum_{d(x, \gamma x) \leq T} e^{-\alpha d(x, \gamma x)}=0$.

Proof. We denote a point of $S H$ by $v=(\zeta, \eta, t), \zeta, \eta \in \partial H$ and $t \in \mathbb{R}$. For any subset $O \subset S H$ and $\zeta \in \partial H$ we denote $O_{\zeta}=O \cap W^{u}(\zeta)$, where $W^{u}(\zeta)=$ $\{v \in S H \mid v(-\infty)=\zeta\}$. Consider the measure $d \mu_{\zeta}=e^{\alpha \beta_{x}(\zeta, \eta)} d \sigma_{x}(\eta) d s$ on $O_{\zeta}$. Let $O$ be the unit tangent bundle of the geodesic ball $B(x, R)$. For $\zeta \in \partial H$ and $T>0$ consider the function

$$
F(T, \zeta)=\frac{1}{T} \mu_{\zeta}\left[g^{T}\left(O_{\zeta}\right) \cap \underset{d(x, \gamma x) \leq T}{\bigcup} \gamma(0)\right] / \mu_{\zeta}\left(O_{\zeta}\right) .
$$

It satisfies

$$
F(T, \zeta) \leq \frac{1}{\mu_{\zeta}\left(O_{\zeta}\right)} \int_{O_{\zeta}}\left[\frac{1}{T} \int_{0}^{T+2 R} \chi_{\Gamma O}\left(g^{t} v\right) d t\right] d \mu_{\zeta}(v) .
$$

According to Lemma (5.4.1), the integrand tends to zero as $T \rightarrow \infty$ for $\mu^{\sigma_{-}}$ almost all $v \in S M$. By the bounded convergence theorem, $\lim _{T \rightarrow \infty} F(T, \zeta)=0$ for almost all $\zeta \in \partial H$. On the other hand, we have:

$$
\begin{aligned}
F(T, \zeta) & =\frac{1}{T} \sum_{d(x, \gamma x) \leq T} \frac{\mu_{\zeta}\left(g^{-T}\left(\gamma O \cap O_{\zeta}\right)\right)}{\mu_{\zeta}\left(O_{\zeta}\right)} \\
& =\frac{1}{T \mu_{\zeta}\left(O_{\zeta}\right)} \sum_{d(x, \gamma x) \leq T} \int_{O_{\zeta}} \chi_{g^{-T}(\gamma O)}(v) e^{\alpha \beta_{x}(\zeta, \eta)} d \sigma_{x}(\eta) d s .
\end{aligned}
$$

If in the last expression we only consider those $\gamma \in \Gamma$ such that $\gamma x$ is close to the antipodal $\zeta^{\prime}$ of $\zeta$ with respect to $x$, then $e^{\alpha \beta_{x}(\zeta, \eta)}$ is bounded from above and below and the same is true for the arc length contribution to the measure $\mu^{\sigma}$. Thus for $\epsilon>0$ there exists a constant $C_{1}=C_{1}(\epsilon)$ such that

$$
F(T, \zeta) \geq C_{1} \frac{1}{T \mu_{\zeta}\left(O_{\zeta}\right)} \sum_{\substack{\gamma \in \Gamma \\ d(x, \gamma x) \leq T,\left|\gamma x-\zeta^{\prime}\right|_{x} \leq \epsilon}} \sigma_{x}\left(O_{\zeta}(\gamma x, R)\right),
$$

where $O_{\zeta}(\gamma x, R)$ is the shadow from $\zeta$ to $\partial H$ of $B(\gamma x, R),|\cdot|_{x}$ is the Gromov distance. On the other hand, we can choose $0<R_{1}<R_{2}$ such that if $R_{1} \leq R \leq R_{2}$ then we have $O_{x}\left(\gamma x, R_{1}\right) \subset O_{\zeta}(\gamma x, R) \subset O_{x}\left(\gamma x, R_{2}\right)$. We can also choose $R_{1}$ such 
that if $R \geq R_{1}$ then one can apply Lemma (3.4.1) to $O_{x}(\gamma x, R)$. Hence by the shadow lemma we obtain

$$
F(T, \zeta) \geq \frac{C_{2}}{T \mu_{\zeta}\left(O_{\zeta}\right)} \sum_{\substack{\gamma \in \Gamma \\ d(x, \gamma x) \leq T,\left|\gamma x-\zeta^{\prime}\right|_{x} \leq \epsilon}} e^{-\alpha d(x, \gamma x)},
$$

for some constant $C_{2}=C_{2}(R, \epsilon)$. Since $\lim _{T \rightarrow \infty} F(T, \zeta)=0$, we get

$$
\lim _{T \rightarrow \infty} \sum_{\substack{\gamma \in \Gamma \\ d(x, \gamma x) \leq T,\left|\gamma x-\zeta^{\prime}\right|_{x} \leq \epsilon}} e^{-\alpha d(x, \gamma x)}=0 .
$$

Since $L(\Gamma)$ is covered by a finite number of balls of radius $\epsilon$ centered at points $\zeta^{\prime}$ antipodal to points $\zeta$, we finish the proof of Theorem (5.4.2).

Theorem (5.4.3). If there is a constant A such that the Poincaré series satisfies

$$
\sum_{\gamma \in \Gamma} e^{-s d(x, \gamma x)} \geq \frac{A}{s-\delta}
$$

for $s>\delta=\delta(\Gamma)$, then $\mu^{\sigma}(S M)<\infty$.

Proof. Denote $n_{k}=\#\left\{\gamma \in \Gamma \mid k-\frac{1}{2}<d(x, \gamma x) \leq k+\frac{1}{2}\right\}$. Then by Theorem (3.6.1), $n_{k} \leq C_{1} e^{k \delta}$. Thus for $s>\delta$ and some constant $C_{2}$, we have

$$
\sum_{k=T+1}^{\infty} n_{k} e^{-s k} \leq \frac{C_{2} e^{T(\delta-s)}}{s-\delta}
$$

If the assumption of the theorem is satisfied, we can choose $T_{0}$ so large that if $T \geq T_{0}$ then $\frac{C_{2}}{T e^{T(s-\delta)}}<\frac{A}{2}$. Therefore for $T \geq T_{0}$ we have

$$
\frac{1}{T} \sum_{\substack{\gamma \in \Gamma \\ d(\gamma x, x) \leq T}} e^{-s d(x, \gamma x)} \geq \frac{A}{(s-\delta) T}-\frac{C_{2} e^{T(\delta-s)}}{T(s-\delta)} \geq \frac{A}{2 T(s-\delta)} .
$$

Now consider a decreasing sequence $\delta_{n} \rightarrow \delta$ and define $T_{n}=\frac{1}{\delta_{n}-\delta}$. We have for $n$ large enough

$$
\frac{1}{T_{n}} \sum_{d(x, \gamma x) \leq T_{n}} e^{-\delta_{n} d(x, \gamma x)} \geq \frac{A}{2} .
$$

However, since $\delta_{n}>\delta$, we also have

$$
\frac{1}{T_{n}} \sum_{d(x, \gamma x) \leq T_{n}} e^{-\delta d(x, \gamma x)}>\frac{A}{2} .
$$

Combining the above estimates and Theorem (5.4.2) we obtain the proof of Theorem (5.4.3).

Corollary (5.4.4). Under the above assumptions and notation, if $n_{k} \geq C e^{\delta k}$ for some constant $C$ then $\mu^{\sigma}(S M)<\infty$.

Proof. For $s>\delta$, We have $\sum_{\gamma \in \Gamma} e^{-s d(x, \gamma x)} \sim \sum_{k \geq 1} n_{k} e^{-s k} \geq C \sum_{k \geq 1} e^{(\delta-s) k} \geq$ $\frac{C}{1-e^{(\delta-s) k}} \geq \frac{C}{s-\delta}$. 
(5.5) Hausdorff dimension and finiteness. Corollary 3.5.3 tells us that the Hausdorff dimension $H D\left(L^{r}(\Gamma)\right)$ of the radial limit set is always $\leq \delta(\Gamma)$. We prove in this section that if a Fuchsian group has finite Bowen-Margulis measure, then $H D\left(L^{r}(\Gamma)\right)=\delta(\Gamma)$.

Theorem (5.5.1). Let $\sigma$ be the $\delta(\Gamma)$-conformal density of a Fuchsian group $\Gamma$ as constructed in (3.2). If $\mu^{\sigma}(S M)<\infty$, then $H D\left(L^{r}(\Gamma)\right)=\delta(\Gamma)$.

We will need the following two lemmas.

Lemma (5.5.2). Fix $w \in S M$. Let $w(0)=x$ and $\sigma(t, v, w)=d(v(t), w(0))$ for $v \in S M$. If $\mu^{\sigma}(S M)<\infty$, then for $\mu^{\sigma}$-almost all $v \in S M, \lim _{t \rightarrow \infty} \frac{1}{t} \sigma(t, v, w)=0$.

Proof. Let $\tau(v)$ be the directional derivative of $\sigma(t, v, w)$ along $v(t)$ at $t=0$. Then $|\tau(v)| \leq 1$ for all $v \in S M$ and since $\mu^{\sigma}(S M)<\infty, \tau(v)$ is integrable. Notice that $\tau(v)=-\tau(-v)$ and that the measure $\mu^{\sigma}$ is flip-invariant. So we get $\int_{S M} \tau(v) d \mu^{\sigma}=0$. By ergodicity we have

$$
\begin{aligned}
\lim _{T \rightarrow \infty} \frac{1}{T}[\sigma(T, v, w)-\sigma(0, v, w)] & =\lim _{T \rightarrow \infty} \frac{1}{T} \int_{0}^{T} \tau\left(g^{t} v\right) d t \\
& =\int_{S M} \tau(v) d \mu^{\sigma}=0 .
\end{aligned}
$$

Lemma (5.5.3). If $\mu^{\sigma}(S M)<\infty$ then there exists a compact set $K \subset L^{r}(\Gamma)$ such that $\sigma_{x}(K)>0$ and moreover, $\forall \epsilon>0$, there exist $C=C(\epsilon)$ and $r_{0}>0$ such that if $\zeta \in K$ and $0<r<r_{0}$, then $\sigma_{x}(B(\zeta, r)) / r^{\delta-\epsilon} \leq C$, where $B(\zeta, r)$ is the metric ball in $\partial H$ with respect to the geodesic metric $d_{x}=d_{x}^{1}$.

Proof. Given $t>0$ and $\zeta \in \partial H$ we define $v(t)$ to be the geodesic ray from $x$ to $\zeta$ and set

$$
D_{t, \zeta}=\left\{y \in \bar{H} \mid \text { the angle between } \overrightarrow{v(t) y} \text { and } \overrightarrow{x v(t)} \text { is } \geq \frac{\pi}{2}\right\} .
$$

By the law of cosine ([BGS], see also Corollary 1.1.4 of [B]), there exists a constant $C_{1}=C_{1}\left(K_{1}, K_{2}\right)$ such that for all $y \in D_{t, \zeta}, e^{d(x, y)} \geq C_{1} e^{d(v(t), y)+t}$. Let $\gamma_{0} x$ be the closest $\Gamma$-image of $x$ to $v(t)$. Then by the triangle inequality, $d(v(t), \gamma x) \geq$ $d\left(\gamma_{0} x, \gamma x\right)-d(v(t), \gamma x)$. Thus for $s>\delta$, we have

$$
\begin{aligned}
e^{s t} \sum_{\gamma \in \Gamma, \gamma x \in D_{t, \zeta}} e^{-s d(x, \gamma x)} & \leq C_{1}^{-s} \sum_{\gamma x \in D_{t, \zeta}} e^{-s d(v(t), \gamma x)} \\
& \leq C_{1}^{-s} e^{s d\left(v(t), \gamma_{0} x\right)} \sum_{\gamma x \in D_{t, \zeta}} e^{-s d\left(\gamma_{0} x, \gamma x\right)} \\
& \leq C_{1}^{-s} e^{s d\left(v(t), \gamma_{0} x\right)} \sum_{\gamma \in \Gamma} e^{-s d(x, \gamma x)} .
\end{aligned}
$$

Let $\sigma_{x}^{s}$ be the measure $\frac{\sum_{\gamma \in \Gamma} e^{-s d(x, \gamma x)} D(\gamma x)}{\sum_{\gamma \in \Gamma} e^{-s d(x, \gamma x)}}$, where $D$ denotes the Dirac measure. Then $(* *)$ implies that $\sigma_{x}^{s}\left(D_{t, \zeta}\right) \leq C_{1}^{-s} e^{-s t} e^{s d\left(\gamma_{0} x, v(t)\right)}$. Letting $s \rightarrow \delta(\Gamma) \triangleq \delta$, we obtain that $\sigma_{x}\left(D_{t, \zeta} \cap \partial H\right) \leq C_{1}^{-\delta} e^{-\delta t} e^{\delta d\left(\gamma_{0} x, v(t)\right)}$. In the case of constant curvature, it is easy to see that there exists a constant $C_{2}=C_{2}\left(K_{1}, K_{2}\right)>0$ such that for all $\eta$ on the boundary of $D_{t, \zeta} \cap \partial H, d_{x}(\zeta, \eta) \geq C_{2} e^{-t}$. In the case of variable negative 
curvature we can still prove prove a similar estimate by using the comparison theorem. See, for example, Lemma 2.4.2 of [B], which ensures the existence of a point $p$ on the geodesic $(\zeta, \eta)$ such that $d(v(t), p) \leq C=C\left(\frac{\pi}{2}, K_{1}, K_{2}\right)$. Thus the distance from $x$ to $(\zeta, \eta)$ satisfies $D_{x}(\zeta, \eta) \leq d(x, p) \leq d(x, v(t))+d(v(t), p) \leq t+C$, which in turn implies an analogous estimate as in the constant curvature case. Writting $r=C_{2} e^{-t}$ we have

$$
\sigma_{x}(B(\zeta, r)) \leq C_{1}^{-\delta}\left(\frac{r}{C_{2}}\right)^{\delta} e^{\delta d\left(\gamma_{0} x, v(t)\right)}=\left(C_{1} C_{2}\right)^{-\delta} e^{\delta d\left(\gamma_{0} x, v(t)\right)} r^{\delta}
$$

By Lemma (5.5.2), given any $\varepsilon>0$, there exist $t_{0}>0$ and a compact set $K \subset L^{r}(\Gamma)$ such that $\sigma_{x}(K)>0$ and such that for all $t \geq t_{0}$ and $\zeta \in K, d\left(\gamma_{0} x, v(t)\right) \leq \varepsilon t$, where $v(t)$ is the geodesic from $x$ to $\zeta$. This finishes the proof of Lemma (5.5.3). The proof of Theorem (5.5.1) is standard once we have Lemma (5.5.3).

(5.6) Ergodicity and recurrence for the harmonic measure. Recall that a Riemannian manifold $M$ is said to be recurrent if almost every Brownian path starting from any point in $M$ enters every open set infinitely often, or equivalently, $M$ admits no Green function. A similar notion of recurrence can be stated for any diffusion process on $M$. First let us recall the following

Theorem (5.6.1) (Ancona $[\mathrm{A} 2])$. Let $\Gamma$ be a Fuchsian group and $M=H / \Gamma$. Let $\nu_{x}$ be the harmonic measure at $x \in H$ on $\partial H$ or on $S_{x} M$. The following properties are equivalent

i) $M$ is recurrent.

ii) For each $x \in M$ and $\nu_{x}$-almost all $v \in S_{x} M$, the image of the geodesic ray $v(t)_{t \geq 0}$ is dense in $M$.

iii) $\nu_{x}\left(L^{\bar{r}}(\Gamma)\right)=1$.

iv) The action of $\Gamma$ on $\partial H \times \partial H$ is ergodic with respect to the measure $\nu_{x} \times \nu_{x}$.

Let $\lambda=\lambda(\Gamma)$ be the first eigenvalue of the Laplacian on $M=H / \Gamma$ and $\sigma$ a $\lambda$-harmonic density of $\Gamma$. If $\lambda<\lambda_{1}$ then such a $\sigma$ exists by (4.2). From now on we assume $\lambda<\lambda_{1}$ and denote by $d \Lambda^{\sigma}$ the $\Gamma$-invariant measure on $\partial H \times \partial H$ constructed in Proposition (5.2.2). We also denote by $d \nu^{\sigma}=d \Lambda^{\sigma} d t$ the corresponding invariant measure on $S M$ of the geodesic flow.

Theorem (5.6.2) (Dichotomy). The geodesic flow $g^{t}$ on $\left(S M, \nu^{\sigma}\right)$ is either

i) completely dissipative (i.e., the random geodesic eventually leaves every compact set forever), or

ii) completely conservative and ergodic. In this case the $\lambda$-harmonic density is unique.

Proof. The conservative dichotomy follows from Theorem (4.3.2). Ergodicity is treated in the following theorem. Uniqueness follows from Proposition (4.2.2).

Theorem (5.6.3). Assume that the Fuchsian group $\Gamma$ satisfies $\lambda(\Gamma)<\lambda_{1}$. Let $\nu^{\sigma}$ be the $g^{t}$-invariant harmonic measure corresponding to a $\lambda \triangleq \lambda(\Gamma)$-harmonic density $\sigma$. Then the following properties are equivalent.

i) $\sigma_{x}\left(L^{r}(\Gamma)\right)=1$.

ii) $\sigma_{x}\left(L^{r}(\Gamma)\right)>0$.

iii) The series $\sum_{\gamma \in \Gamma} G_{\lambda}(\gamma x, y)$ diverges for $y \in H \backslash \Gamma x$.

iv) The geodesic flow is ergodic with respect to $\nu^{\sigma}$. 
Proof. The equivalence i) $\Leftrightarrow$ ii) follows from Theorem (4.3.2). To prove iii) $\Rightarrow$ iv): Recall the $\Phi$-process in the proof of Theorem (4.3.2). The following argument of Sullivan ([S1]) for the constant curvature case works just as well for the variable curvature case. The divergence of the series $\sum_{\gamma \in \Gamma} G_{\lambda}(\gamma x, y)$ implies that the quotient $\Phi$-process on $M$ is recurrent. Then the recurrence property for the quotient $\Phi$-process implies that combined action of $\Gamma$ and time shift on the space of biinfinite paths of the $\Phi$-process is ergodic, which easily implies the ergodicity of the geodesic flow on $\left(S M, \nu^{\sigma}\right)$. To prove i) $\Rightarrow$ iii): If the series $\sum_{\gamma \in \Gamma} G_{\lambda}(\gamma x, y)$ converges then it defines a Green function for the operator $L$ (see Theorem (4.3.2)) on $M$. Thus the $\Phi$-process is transient, which contradicts i). Finally, the implication iv) $\Rightarrow$ i) is obvious.

Corollary (5.6.4). If $\Gamma$ has a fundamental domain $D$ in $H$ with $\bar{D} \cap L(\Gamma)=\varnothing$, then the geodesic flow is ergodic with respect to $\nu^{\sigma}$.

Proof. This follows from Theorem (4.2.1).

\section{Geometrically finite groups And CONVEX COCOMPaCt GRoups}

(6.1) Definitions and basic properties. We continue to assume that $\Gamma$ is a Fuchsian group of a simply connected Riemannian manifold of pinched negative curvature. Let $L(\Gamma)$ be the limit set and $O(\Gamma)=\partial H \backslash L(\Gamma)$ be the ordinary set. The Kleinian manifold associated to $\Gamma$ is defined to be $\bar{M}_{\Gamma}=H \cup O(\Gamma) / \Gamma$. The Gromov geodesic hull of $\Gamma$ is defined to be $G(\Gamma)=(L(\Gamma) \times L(\Gamma) \backslash$ diagonal $) \times R$, i.e., the union of all geodesics $\gamma$ in $H$ with $\gamma(-\infty), \gamma(\infty) \in L(\Gamma)$. Generally, $G(\Gamma)$ is not a convex set. But it is a subset of the convex hull $H(\Gamma) \triangleq$ the smallest convex set in $\bar{H}$ containing $L(\Gamma)$. It is well-known that $\overline{G(\Gamma)} \cap \partial H=L(\Gamma)$ and that $G(\Gamma)$ is an $\varepsilon$-convex set for some $\varepsilon>0$; i.e., for all geodesic segments $\gamma:[a, b] \rightarrow H$ with $\gamma(a), \gamma(b) \in G(\Gamma)$, one has $d(\gamma(t), G(\Gamma)) \leq \varepsilon$ for all $a \leq t \leq b$ (Gromov [G]). Let $p \in L(\Gamma)$ be a parabolic fixed point and let $\Gamma_{p}$ be the maximal parabolic subgroup of $\Gamma$ fixing $p$.

Definition (6.1.1). A parabolic fixed point $p \in L(\Gamma)$ is said to be bounded if $(L(\Gamma) \backslash\{p\}) / \Gamma_{p}$ is compact. An end of the Kleinian manifold $\bar{M}_{\Gamma}$ is called a parabolic end if it is associated to a bounded parabolic point.

Theorem (6.1.2). The following properties are equivalent (see $[\mathrm{B}])$ :

i) $\bar{M}_{\Gamma}$ has finitely many ends, each a parabolic end.

ii) $L(\Gamma)=L^{r}(\Gamma) \cup\{$ bounded parabolic fixed points $\}$.

iii) $H(\Gamma) / \Gamma \cap$ thick $(M)$ is compact for some $0<\varepsilon<\varepsilon\left(K_{1}, K_{2}\right)$, where $\varepsilon\left(K_{1}, K_{2}\right)$ is the Margulis constant and $\operatorname{thick}_{\varepsilon}(M)=\{x \in M \mid \operatorname{inj}(x, M) \geq \varepsilon / 2\}$.

iv) For some $\varepsilon>0$, the $\varepsilon$-neighborhood of $H(\Gamma) / \Gamma$ in $M$ has finite volume.

Definition (6.1.3). A Fuchsian group $\Gamma$ is called geometrically finite if it satisfies any one of the above properties.

Definition (6.1.4). $\quad$ i) $\Gamma$ is said to be geodesic cocompact if $G(\Gamma) / \Gamma$ is compact.

ii) $\Gamma$ is said to be convex cocompact if $H(\Gamma) / \Gamma$ is compact.

It is easy to see that a convex cocompact group is always geometrically finite. A geometrically finite group is convex cocompact if and only if it has no parabolic elements. 
Proposition (6.1.5). A Fuchsian group $\Gamma$ is convex cocompact if and only if it is geodesic cocompact.

Proof. Since the Gromov geodesic hull $G(\Gamma)$ is always a subset of the convex hull $H(\Gamma)$ of $\Gamma$, hence we have the easy implication that convex cocompact $\Rightarrow$ geodesic cocompact. By a theorem of Anderson ([And]), for any compact subset $W$ in the ideal boundary of a Hadamard manifold $H$ with pinched negative curvature, the geodesic hull $G(W)$ admits a convex neighborhood in $H$ lying within a bounded distance from $G(W)$. It follows that geodesic cocompact $\Rightarrow$ convex cocompact.

Proposition (6.1.6). If $\Gamma$ is convex cocompact, then we have:

(i) $L^{r}(\Gamma)=L(\Gamma)$.

(ii) Let $D$ be any convex fundamental domain of $\Gamma$ in $H$ (for example, the Dirichlet domain). Then $\bar{D} \cap L(\Gamma)=\varnothing$.

Proof. (i) Assume that $\zeta \in L(\Gamma)$. Let $\gamma(t)$ be a geodesic satisfying $\gamma(\infty)=\zeta$ and $\gamma(-\infty) \in L(\Gamma)$. Then $\gamma \subset G(\Gamma)$. Let $D$ be a compact fundamental domain of $\Gamma$ acting on $G(\Gamma)$. Then there exists a sequence $\left\{\gamma_{n}\right\}_{n \geq 0} \subset \Gamma$ such that $\gamma \subset \cup_{n \geq 0} \gamma_{n} D$. This implies that $\zeta$ is a radial limit point.

(ii) Assume that $\zeta \in \bar{D} \cap L(\Gamma)$. Since $D$ is convex, there exists a geodesic ray $\sigma$ joining $x$ in $D$ to $\zeta$. Since $\zeta$ is a radial limit point $((\mathrm{i})), \gamma_{n}(\sigma) \cap K \neq \varnothing$ for a infinite sequence of $\gamma_{n} \in \Gamma$ and a compact set $K \subset H$, which contradicts the proper discontinuity of $\Gamma$.

The following theorem exhibits a fine property of convex cocompact groups.

Theorem (6.1.7) $([\mathrm{C}])$. Any convex cocompact Fuchsian group $\Gamma$ is a hyperbolic group in the sense of Gromov.

(6.2) The conformal density of a convex cocompact group as a Hausdorff measure and a lattice counting formula. Suppose that $\Gamma$ is convex cocompact. By Proposition (6.1.6), $L^{r}(\Gamma)=L(\Gamma)$. Thus according to Corollary (3.5.6), we have:

i) $\Gamma$ has a unique conformal density $\sigma$ of dimension $\delta=\delta(\Gamma)$ and the $\Gamma$ action on $L(\Gamma)$ is ergodic with respect to $\sigma$.

ii) $\Gamma$ is of divergent type, i.e., $\sum_{\gamma \in \Gamma} e^{-\delta(\Gamma) d(x, \gamma x)}=\infty$.

The following theorems, which were proved in [C] for hyperbolic groups, are clearly true under our set up.

Theorem (6.2.1) ([C]). Let $B(\zeta, r)$ be the ball on $\partial H$ with respect to any one of the four metrics defined in section 1 . There exists a constant $C \geq 1$ such that for all $\zeta \in L(\Gamma)$ and $r \geq 0$,

$$
C^{-1} r^{\delta} \leq \sigma_{x}(B(\zeta, r)) \leq C r^{\delta}
$$

Corollary (6.2.2) $([\mathrm{C}])$. Let $\mathcal{H}^{\delta}$ be the $\delta$-dimensional Hausdorff measure with respect to any one of the above four metrics. There exists a constant $C \geq 1$ such that for all $A \subset L(\Gamma)$,

$$
C^{-1} \mathcal{H}^{\delta}(A) \leq \sigma_{x}(A) \leq C \mathcal{H}^{\delta}(A) .
$$

The following theorem shows that the $\delta(\Gamma)$-conformal density of a convex cocompact group $\Gamma$ is exactly the $\delta$-dimensional Hausdorff measure associated with the Busemann metric. 
Theorem (6.2.3). The $\delta$-dimensional Hausdorff measure $\mathcal{H}_{B_{x}}^{\delta}$ with respect to the Busemann metric $B_{x}$ gives rise to a $\delta$-conformal density of $\Gamma$ and $\mathcal{H}_{B_{x}}^{\delta}=K \sigma_{x}$ for some constant $K$.

Proof. This follows from Proposition (3.1.1) and the uniqueness of the $\delta(\Gamma)$-dimensional conformal density for a convex cocompact group.

In [C], Coornaert also proved the following result.

Theorem (6.2.4)([C]). Let $n_{x}(R)=\#\{\Gamma x \cap B(x, R)\}$. Then there exists a constant $C \geq 1$ such that

$$
C^{-1} e^{\delta R} \leq n_{x}(R) \leq C e^{\delta R}
$$

We provide an exact formula in the following

Theorem (6.2.5). Let $\Gamma$ be a convex cocompact group. Let $\sigma$ be the $\delta(\Gamma)$-conformal density and $c(x)=\sigma_{x}(\partial H)$. Let $N(x, y, R)=\#\{\Gamma x \cap B(y, R)\}$. Then there is a constant $C>0$ such that for all $x, y \in H$,

$$
N(x, y, R) \sim C c(x) \cdot c(y) e^{\delta R} .
$$

Proof. In his thesis ([M, Theorem 8, p.99]), Margulis proved that if $\Gamma$ is cocompact, then

$$
N(x, y, R) \sim \frac{\tilde{\mu}_{S_{x} M}\left(S_{x} M\right) \tilde{\mu}_{S_{y} M}\left(S_{y} M\right)}{\mu(S M)} \int_{0}^{R} e^{h t} d t,
$$

where $h$ is the topological entropy, $\mu$ is the Bowen-Margulis measure, $\tilde{\mu}_{S_{x} M}$ is a measure he constructed via the following process ([M] p. 83-85):

Let $\mathcal{U}_{k}=\bigcup_{i=1}^{n(k)} P_{i}^{(k)}$ be a finite open cover of the unit tangent sphere $S_{x} M$. Each $P_{i}^{(k)}$ has a $W^{s u} \times W^{s}$-local product structure with diameter $\leq \frac{1}{k}$. Then by sliding the Margulis measure on a close $W^{s u}$-piece along $W^{s}$ leaves to $S_{x} M$ (taking care of the intersections) we obtain a measure $\mu_{k}$ on $S_{x} M$. Then any weak limit of $\left\{\mu_{k}\right\}$ as $k \rightarrow \infty$ gives us the measure $\tilde{\mu}_{S_{x} M}$. Now assume that we are in the convex cocompact case and recall the Margulis measure along $W^{s u}$-leaves we constructed from a conformal density (see $(5.2 .0 *)$ ). We can perform a construction similar to the above cocompact case to obtain measures on unit tangent spheres. Namely, we can choose a finite open cover $\mathcal{U}_{k}$ of the unit tangent sphere at $x$. It is easy to see that if the diameter of the sets in $\mathcal{U}_{k}$ is small, then the measure $\mu_{k}$ obtained by sliding as in the cocompact case is close to the conformal density $\sigma_{x}$. Consequently, by the uniqueness of the conformal density and the uniqueness of the Bowen-Margulis measure for convex-cocompact groups, and also by the comment in front of Proposition (5.2.2), we conclude that the limit measure $\tilde{\mu}_{S_{x} M}$ of the measures $\mu_{k}$ satisfies $\tilde{\mu}_{S_{x} M}=C \cdot \sigma_{x}$, where $C$ is a constant which does not depend on $x$. In the cocompact case we get $N(x, y, R) \sim C \cdot c(x) c(y) e^{h R}$, where $c(x)$ is the Margulis function $c(x)=\lim _{R \rightarrow \infty} \frac{\operatorname{Vol}(S(x, R))}{e^{h R}}$ (it seems that Margulis did not realize this fact in his thesis). Margulis's argument in the cocompact case depends only on the local decomposition properties $(5.2 .1 *)-(5.2 .3 *)$ and the fact that the Bowen-Margulis measure is mixing. In the convex cocompact case, both properties are still satisfied(see the next section for the mixing property) and all of Margulis's arguments still apply. Hence we have an analogous formula. 
(6.3) Gibbs measures. We continue to assume $\Gamma$ to be a convex cocompact group. This is equivalent to saying that the nonwandering set $\Omega$ of the geodesic flow $g^{t}$ on $S M=S(H / \Gamma)$ is compact. In the terminology of dynamical systems, the nonwandering set is a compact hyperbolic invariant set and the geodesic flow satisfies Axiom A. Moreover, by Proposition (5.1.2), $\left.g^{t}\right|_{\Omega}$ is topologically transitive with dense periodic vectors. Thus the whole theory of thermodynamic formalism applies here. In particular, for each Hölder continuous function $\varphi: \Omega \rightarrow \mathbb{R}$, there exists a unique probability measure $\mu_{\varphi}$ on $\Omega$ (the so-called equilibrium state of the function $\varphi$ ), which is invariant and ergodic under the geodesic flow and is positive on non-empty open sets of $\Omega$. If we denote by $P_{\varphi}$ the topological pressure of $\varphi$ and by $h_{\mu}$ the metric entropy of an invariant measure $\mu$. Then we have

$$
P_{\varphi}=\sup _{\mu \in M\left(g^{t}\right)}\left\{h_{\mu}(f)+\int \varphi d \mu\right\},
$$

where $M\left(g^{t}\right) \triangleq\left\{\right.$ all $g^{t}$-invariant probability measures on $\left.\Omega\right\}$. The supremum is achieved uniquely by a measure $\mu_{\varphi}$ which is called the Gibbs measure of the potential function $\varphi$. The Gibbs measures satisfy nice properties. For example, for any $\varepsilon>0$, there exists $C_{\varepsilon}>0$ so that for all $v \in \Omega$ and $T \geq 0$,

$$
\mu_{\varphi}\left(B_{v}(\varepsilon, T)\right) \geq C_{\varepsilon} e^{-\left[P_{\varphi}+\int_{0}^{T} \varphi\left(g^{t} v\right) d t\right]},
$$

where $B_{v}(\varepsilon, T)=\left\{w \in \Omega \mid d\left(g^{t} w, g^{t} v\right) \leq \varepsilon\right.$ for all $\left.0 \leq t \leq T\right\}$.

Theorem (6.3.1). Let $\sigma$ be the $\delta=\delta(\Gamma)$ conformal density of $\Gamma$ and let $\mu^{\sigma}$ be the $g^{t}$-invariant measure constructed in (5.2). Then $\mu^{\sigma}$ is the unique equilibrium state of the function $\varphi=0$ up to a scalar multiplication. Moreover, $\mu^{\sigma}$ maximizes the topological entropy of the geodesic flow.

Proof. By Theorem (6.2.4) and Corollary (5.4.4), $\mu^{\sigma}$ is a finite measure on $\Omega$. On the other hand, as we remarked in section (5.2), the measure $\mu^{\sigma}$ can be described as $d \mu^{\sigma}=d \mu^{u} \times d \mu^{s s}=d \mu^{s} \times d \mu^{s u}$, where $\mu^{i}(i=s, u, s s, s u)$ are measures along the Anosov foliation which are uniformly expanding (or contracting) by the geodesic flow. By the uniqueness of the finite Bowen-Margulis measure (i.e., measures having the above local decomposition) on basic hyperbolic sets, $\mu^{\sigma}$ must be the unique measure which maximizes the topological entropy.

By Proposition (6.1.6), the Dirichlet domain $D$ of a convex cocompact group $\Gamma$ satisfies $\bar{D} \cap L(\Gamma)=\varnothing$. According to Corollary (5.6.4), $\Gamma$ has a unique $\lambda=\lambda(\Gamma)$ harmonic density $\sigma$ and the geodesic flow $g^{t}$ is ergodic with respect to the measure $\nu^{\sigma}$.

Theorem (6.3.2). For a convex cocompact group $\Gamma$, we have $\nu^{\sigma}(\Omega)<\infty$. Moreover, the measure $\nu^{\sigma}$ is the unique equilibrium state of the function $\tau: \Omega \rightarrow R$, $\left.\tau(v) \triangleq \frac{d}{d t}\right|_{t=0} K_{\lambda}(v(0), v(t), v(\infty))$, where $K_{\lambda}$ is Poisson kernel we introduced in section (4.1).

Proof. We follow a construction of Ledrappier ([L1]). Compare also (5.2). Fix a point $x \in H$. Using the canonical homeomorphism $W^{s u}(v) \stackrel{P}{\rightarrow} \partial H \backslash\{v(-\infty)\}$, we can pull the measure $\sigma_{x}$ back to $W^{s u}(v)$ (which is denoted by the same symbol) and then define a measure $\nu^{s u}(v)$ on $W^{s u}(v)$ by

$$
\frac{d \nu^{s u}}{d \sigma_{x}}(w)=K_{\lambda}(x, w(0), w(\infty))
$$


The measure $\nu^{s u}$ does not depend on the choice of $x$ and is $\Gamma$ invariant. It transforms under the geodesic flow via

$$
\frac{d \nu^{s u}}{d \nu^{s u} \circ g^{-t}}\left(g^{t} v\right)=K_{\lambda}(v(0), v(t), v(\infty)) .
$$

Similarly one can construct a family of $\Gamma$-invariant measure $\nu^{s s}$ on the $W^{s s}$ leaves which transforms under the geodesic flow via

$$
\frac{d \nu^{s s}}{d \nu^{s s} \circ g^{-t}}\left(g^{t} v\right)=K_{\lambda}(v(0), v(t), v(\infty))^{-1} .
$$

Combining them together we obtain a flow invariant measure $d \nu=d \nu^{s u} \times d \nu^{s s} \times d t$. It can be projected to $S M$ since it is $\Gamma$-invariant.

On the other hand, consider the function $\tau_{0}(v)$ on $S M$ defined by $\tau_{0}(v)=$ $\ln K_{\lambda}(v(0), v(1), v(\infty))$. We claim that $\tau_{0}$ is Hölder continuous. By Theorem 6.2 and Theorem 6.3 in [AS], we see that in order to prove the Hölder regularity of the Poisson kernel $K_{\lambda}(x, y, \zeta)$ as a function of $\zeta$, it suffices to check two things:

1) the Hölder structure of $\partial H$,

2) a Harnack inequality at infinity for the operator $L=\Delta-\lambda I$.

Condition 1) follows naturally from our curvature pinching condition and condition 2 ) is proved in Theorem 2 of [A3]. Now let $\mu$ be any limit point of $\frac{1}{t} \int_{0}^{t} \nu^{s u} \circ g^{-s} d s$ as $s \rightarrow \infty$. According to [Si2], the conditional measures of $\mu$ along the $W^{s u}$ foliation are equivalent to $\nu^{s u}$. From this it follows that $h_{\mu}\left(g_{1}\right)=\int \tau_{0} d \mu$ (see [Si1]). We also have $h_{m}\left(g_{1}\right) \leq \int \tau_{0} d m$ for any invariant probability measure $m$ (see [LY]). Thus $\mu$ is the unique equilibrium state of $\tau_{0}$ and it must coincide with the measure $\nu$. Since $\int_{0}^{1} \tau\left(g^{s} v\right) d s=\tau_{0}(v)$, the function $\tau$ has the same equilibrium state as $\tau_{0}$ and their topological pressure is equal to zero. By the description of the measures $\nu$ and $\nu^{\sigma}$ it is easy to see that they are absolutely continuous with respect to each other. Let $f(v)=\frac{d \nu^{\sigma}}{d \nu}(v)$. Since $f$ is $g_{t}$-invariant and both $\nu^{\sigma}$ and $\nu$ are $g^{t}$-ergodic, $f$ must be a positive constant.

(6.4) Hausdorff dimension of Gibbs measures. We continue to consider a convex cocompact group $\Gamma$. Let $G\left(g^{t}\right)=\{$ all Gibbs measures, i.e., the equilibrium states of all Hölder functions on $\Omega\}$. Let $\mu \in G\left(g^{t}\right)$. For any measurable partition $\zeta$ subordinate to the strong unstable foliation (see $[\mathrm{LY}]$ for definition) there is a canonical family of conditional measures $\mu_{v}^{\zeta}$ on $\zeta(v)$. Via the projection $\zeta(v) \stackrel{P}{\rightarrow}$ $\partial H: w \rightarrow w(\infty), \mu_{v}^{\zeta}$ can be projected to $\partial H$ and by the quasi-invariance of Gibbs measures under the weak stable foliation, we have that for $\mu$-almost all $v, w \in S M$, if $P(\zeta(v)) \cap P(\zeta(w)) \neq \varnothing$, then the measures $P^{*}\left(\mu_{v}^{\zeta}\right)$ and $P^{*}\left(\mu_{w}^{\zeta}\right)$ are equivalent on the subset $P(\zeta(v)) \cap P(\zeta(w))$. Thus they define a measure class on $\partial H$, which we denote by $\partial \mu$.

Definition (6.4.1). Let $\rho$ be any metric on $\partial H$. The $\rho$-Hausdorff dimension of $\partial \mu$ is defined to be

$$
H D_{\rho}(\partial \mu)=\inf _{\substack{A \subset \partial H \\ \partial \mu(\partial H \backslash A)=0}} H D_{\rho}(A) .
$$

Theorem (6.4.2). Let $\mu$ be a Gibbs measure. Let $\eta$ be any one of the four metrics defined in section 1. Then the $\eta$-Hausdorff dimension of $\partial \mu$ is equal to $h_{\mu}$. 
Proof. By a result of L.S. Young ([Y]), it suffices to prove that for $\partial \mu$-almost all $\zeta \in \partial H, \lim _{\epsilon \rightarrow 0} \frac{\log \partial \mu B_{\eta}(\zeta, \varepsilon)}{\log \varepsilon}=h_{\mu}$. By the equivalence of all four metrics and the definition of $\rho_{x}$, it is enough to prove that

$$
\lim _{T \rightarrow \infty} \frac{\log P^{*}\left(\mu_{v}^{\zeta}\right)\left\{w(\infty) \mid w \in S_{x} M, \quad d\left(g^{t} v, g^{t} w\right) \leq 1 \text { for all } 0 \leq t \leq T\right\}}{T}=-h_{\mu},
$$

where $d\left(g^{t} v, g^{t} w\right) \triangleq d(v(t), w(t))$ is the Riemannian distance between the two points $v(t)$ and $w(t)$. According to $[\mathrm{LY}]$,

$$
\lim _{T \rightarrow \infty} \frac{1}{T} \log \mu_{v}^{\zeta}\left\{w \in \zeta(v) \mid d\left(g^{t} v, g^{t} w\right) \leq 1,0 \leq t \leq T\right\}=-h_{\mu}
$$

The proof of the theorem is completed by the following lemma.

Lemma (6.4.3). For any $x \in H, v \in S H$, consider the metric $\rho_{x}$ which is introduced in section 1 , and the metric $\eta_{v}$ on $\partial H \backslash\{v(-\infty)\}$, which is defined by: $\eta_{v}(\zeta, \eta)=e^{-t_{v}(\zeta, \eta)}$, where $t_{v}(\zeta, \eta) \triangleq \sup \left\{t \geq 0 \mid d\left(g^{t} w_{1}, g^{t} w_{2}\right) \leq 1\right\}$ and $w_{1}, w_{2}$ are the unique vectors on $W^{s u}(v)$ satisfying $w_{1}(\infty)=\zeta, w_{2}(\infty)=\eta$. Then there exist a neighborhood $\triangle$ of $v(\infty)$ on $\partial H$ and a constant $C>0$ such that for all $\zeta, \eta \in \triangle$, $C^{-1} \eta_{v}(\zeta, \eta) \leq \rho_{x}(\zeta, \eta) \leq C \eta_{v}(\zeta, \eta)$.

Proof. Assume that $x$ is a point on the horosphere $H^{s u}(v)$ (which is also the projection of $W^{s u}(v)$ to $\left.H\right)$. Then we have the following estimates:

(1) There is a constant $C_{1}>0$, such that for the vector $v_{1} \in S_{x} M$ with $v_{1}(\infty)=$ $\zeta$, we have $d\left(g^{t} w_{1}, g^{t} v_{1}\right) \leq C_{1} e^{-K_{1} t}$, and for the vector $v_{2} \in S_{x} M$ with $v_{2}(\infty)=\eta$, we have $d\left(g^{t} w_{2}, g^{t} v_{2}\right) \leq C_{1} e^{-K_{1} t}$.

(2) There exists a constant $C_{2}>0$, such that $C_{2}{ }^{-1} e^{K_{1} t} \leq d\left(g^{t} w_{1}, g^{t} w_{2}\right) \leq$ $\mathrm{C}_{2} e^{K_{2} t}$.

Assuming that $d\left(g^{t_{0}} v_{1}, g^{t_{0}} v_{2}\right)=1$, we obtain $\left|d\left(g^{t_{0}} w_{1}, g^{t_{0}} w_{2}\right)-1\right| \leq 2 C_{1} e^{-K_{1} t_{0}}$. Combining this and (2) we get the proof of the lemma.

(6.5) Lattices. Unlike the convex cocompact case and the constant curvature case, we do not have a clear picture for the ergodic theory of geometrically finite Fuchsian groups. This is partly due to the fact that the asymptotic geometry along different cusps could behave differently. Let us consider the simplest case when $\Gamma$ is a non-uniform lattice. Then $M=H / \Gamma$ is diffeomorphic to the interior of a compact manifold with boundary. We have a Margulis decomposition $M=M_{0} \cup \bigcup_{i=1}^{k} M_{i}$, where $M_{0}$ is the compact part and $M_{i}=N_{i} \times(0, \infty), N_{i}=L_{i} / \Gamma_{\zeta_{i}}$ with $L_{i}$ a horosphere at $\zeta_{i} \in \partial H$ and $\Gamma_{\zeta_{i}} \subset \Gamma$ a maximal parabolic subgroup fixing $\zeta_{i}$. Each $N_{i}$ is a $C^{2}$-codimension 1 compact submanifold of $M$.

From now on, we make the following assumption $(* * *)$ : for some (and hence for all) $x \in H, \lim _{R \rightarrow \infty} \frac{v(x, R)}{e^{h R}}=c_{1}(x)$ for some constant $h>0$ (here $v(x, R)=$ $\operatorname{Vol}(B(x, R)))$. This condition is satisfied in the following two cases:

(1) $H$ is a symmetric space (in this case $c_{1}(x)$ is constant for all $x$ ).

(2) $H$ has a compact quotient (in this case, $c_{1}(x)$ is a bounded function on $H$ ).

Let $\Gamma$ be any lattice on $H$ satisfying $(* * *)$. Then it is easy to see that $\delta(\Gamma)=h$. Moreover, we have

Theorem (6.5.1). For any Hadamard manifold $H$ of pinched negative curvature satisfying $(* * *)$ and a lattice $\Gamma$ of $H$, the lattice counting function $N(x, y, R)=$ $\#\{\Gamma y \cap B(x, R)\}$ satisfies $N(x, x, R) \geq c e^{h R}$ for some constant $c$. 
Proof. Let $D$ be the Dirichlet region of $\Gamma$ at $x$. Then $D$ consists of a compact part and finitely many cusps. Given $\varepsilon>0$, there exists $r_{0}>0$ such that $\operatorname{Vol}\left(D \backslash D\left(r_{0}\right)\right) \leq \varepsilon$, where $D\left(r_{0}\right)=D \cap B\left(x, r_{0}\right)$. We can also choose $r_{0}$ so that, for $r \geq r_{0}, v(x, r) \geq\left[c_{1}(x)-\varepsilon\right] e^{h r}$. Then for all $R \geq r_{0}$ we have

$$
\begin{aligned}
& v\left[B(x, R) \cap \Gamma\left(D\left(r_{0}\right)\right)\right]=v(x, R)-v\left(B(x, R) \cap \Gamma\left(D \backslash D\left(r_{0}\right)\right)\right) \\
& \geq\left(c_{1}-\varepsilon\right) e^{h R}-\int_{D-D\left(r_{0}\right)} N(R, x, y) d y \geq\left(c_{1}-\varepsilon\right) e^{h R}-c_{2} .
\end{aligned}
$$

The last inequality is due to, according to Theorem (3.6.1), $N(R, x, y) \leq c_{2} e^{h R}$ for some constant $c_{2}>0$. Thus $v\left(D\left(r_{0}\right)\right) \cdot N\left(R+r_{0}, x, x\right) \geq\left(c_{1}-\varepsilon-c_{2} \varepsilon\right) e^{h R}$.

Theorem (6.5.2). Let $H$ be a Hadamard manifold satisfying $(* * *)$, let $\Gamma$ be a discrete isometry group of $H$, and let $\sigma$ be the $\delta(\Gamma)$-conformal density of $\Gamma$. Then we have:

(1) $\mu^{\sigma}(S M)<\infty$.

(2) $g^{t}$ is ergodic with respect to $\mu^{\sigma}$.

(3) The Poincaré series diverges at $\delta=\delta(\Gamma)=h$.

(4) The Hausdorff dimension of $\partial H$ with respect to any one of the four distances in section 1 is $h$.

Proof. This follows from the finiteness critera in Corollary (5.4.4).

Let $S(x, R)$ be the geodesic sphere in $H$ and $S_{x}(R)$ be its volume. If $H$ satisfies $(* * *)$ then it is easy to see that the following limit exists:

$$
\lim _{R \rightarrow \infty} \frac{S_{x}(R)}{e^{h R}}=\frac{c_{1}(x)}{h} \triangleq c(x) .
$$

Now consider the measure $d \sigma_{x}^{R}(y)=\frac{d m_{x}^{R}(y)}{e^{h R}}$ on $S(x, R)$, where $m_{x}^{R}$ is the Riemannian volume on $S(x, R)$. Push the measures $\sigma_{x}^{R}$ to infinity via the projection $S(x, R) \rightarrow \partial H: y \rightarrow v_{x y}(\infty)$, where $v_{x y}(\infty)$ is the point at infinity corresponding to the geodesic ray from $x$ to $y$; we obtain a family of uniformly bounded measures on $\partial H$ which we denote by the same symbol. Let $\tilde{\sigma}_{x}=\lim _{R_{i} \rightarrow \infty} \sigma_{x}^{R_{i}}$ be any weak

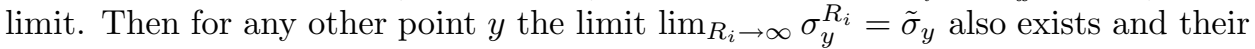
Radon-Nikodym derivative satisfies

$$
\frac{d \tilde{\sigma}_{y}}{d \tilde{\sigma}_{x}}(\zeta)=e^{-h \rho_{x, \zeta}(y)} .
$$

By the uniqueness of $h$-conformal density, $\tilde{\sigma}_{x}$ must coincide with $\sigma$ up to a scalar multiplication. If we assume moreover that $c(x)$ is a bounded (or $L^{1}$ ) function on $M$, then the measure $w^{s s}$ on $S M$ defined by $\int_{S M} \varphi d w^{s s} \stackrel{\text { def }}{=} \int_{M} d x\left(\int_{S_{x} M} \varphi(x, \zeta) d \sigma_{x}(\zeta)\right)$ is a finite measure on $S M$. The results in [Yu1] can be generalized to the finite volume case. In the following theorem we normalize the metric such that $\int_{S M} d w^{s s}=1$.

Theorem (6.5.3). Let $H$ be a Hadamard manifold with pinched negative curvature. If $\lim _{R \rightarrow \infty} \frac{v(x, R)}{e^{h R}}=c(x)$ for some bounded or $L^{1}$ function $c(x)$ on $H$. Then we have

$1^{\circ} . c(x)$ is smooth. 
$2^{\circ}$. If $c(x)$ is a constant function, then for each $x \in H, h=\int_{\partial H} \operatorname{tr} U(x, \zeta) d \bar{\sigma}_{x}(\zeta)$, where $\bar{\sigma}_{x}$ is the normalized Patterson-Sullivan measure and $\operatorname{tr} U(x, \zeta)$ is the mean curvature at $x$ of the horosphere $H^{s s}(x, \zeta)$ passing through $x$ and with center $\zeta$.

$3^{\circ} . h=\int_{S M} \operatorname{tr} U d w^{s s}$.

$4^{\circ} . h^{2}=\int_{S M}\left(R^{H}(v)-R(v(0))+\operatorname{Ric}(v)\right) d w^{s s}$, where $R(v(0))$ is the scalar curvature of $M$ at $v(0), \operatorname{Ric}(v)$ is the Ricci curvature, $R^{H}(v)$ is the scalar curvature of the horosphere $H^{s s}(v)$.

$5^{\circ}$. If $\operatorname{dim} M=3$, then $h^{2}=\int_{S M}(\operatorname{Ric}(v)-R(v(0))) d w^{s s}$.

$6^{\circ}$. If $\operatorname{dim} M=2$, then $h^{2}=\int_{M} c(x) K(x) d m(x)$, where $K(x)$ is the sectional curvature and $d m$ is the Riemannian volume.

On the other hand, without any extra global assumption, we have a fine ergodic theory for the harmonic measure.

Theorem (6.5.4). Let $\Gamma$ be a lattice and $\sigma_{x}$ be the unique harmonic measure on $\partial H$. Let $\nu^{\sigma}$ be the measure on $S M$ we constructed in Proposition (5.2.2). Then the geodesic flow is ergodic with respect to $\nu^{\sigma}$.

Proof. According to Theorem (6.1.2) ii), $\partial H=L^{r}(\Gamma) \cup$ \{bounded parabolic fixed points\}. Since the set of all parabolic fixed points of $\Gamma$ on $\partial H$ is countable and the harmonic measure $\sigma_{x}$ has no atom $([\mathrm{KL}])$, the $\sigma_{x}\left(L^{r}(\Gamma)\right)$ has full measure. Hence $\nu^{\sigma}$ must be ergodic by Theorem (5.6.3).

Next we give a proof of Theorem G in the introduction. Actually Ratner's argument in $[\mathrm{R}]$ for the $S L(2, R)$ case can be translated verbatim as soon as the invariant (after a reparametrization) measure $\mu^{\sigma}$ of the horocycle flow is finite, plus the following observations:

(1) Property (1.2) of [R] is ensured by the corresponding property of the Margulis conditional measure (see section 5.2).

(2) The orbit closure property of the horocycle flow follows from Theorem 5.5 of [E2].

(3) For the proof of uniform distribution of horocycle orbits, one needs the property that if $h^{t} v$ is not a closed orbit, then there exists a compact set $K \subset S M$ and a sequence $t_{n} \rightarrow \infty$ such that $g^{t_{n}} v \in K$ for all $n=1,2, \ldots$ This is equivalent to the following fact: if $v(\infty)$ is not a parabolic fixed point, then it is a radial limit point. This fact is guaranteed by our Theorem (6.1.2) ii). Compare also the proof of Theorem (6.5.4) and the discussion there.

(6.6) Bottom of spectrum, the connection between $\delta(\Gamma)$ and $\lambda(\Gamma)$. Let $\Gamma$ be any Fuchsian group and let $\triangle_{M}$ be the Laplacian operator on $L^{2}(M)$. Then the bottom spectrum $\lambda(\Gamma)$ of the Laplacian $\triangle_{M}$ can be described as the maximum of all $\lambda$ such that $\left(\triangle_{M}+\lambda\right)^{-1}$ converges. This is equivalent to saying that the kernel $g_{\lambda}(x, y)=\int_{0}^{\infty} e^{t \lambda} H_{t}(x, y) d t$ converges for $x \neq y$, where $H_{t}(x, y)=\sum_{\gamma \in \Gamma} P_{t}(\hat{x}, \gamma \hat{y})$ and $P_{t}$ is the heat kernel in $H$ and $\hat{x}, \hat{y}$ are lifts of $x, y$ in $H$. Since the Green function $G_{\lambda}$ of the operator $L=\triangle+\lambda$ in $H$ is equal to $G_{\lambda}(\hat{x}, \hat{y})=\int_{0}^{\infty} e^{t \lambda} P_{t}(\hat{x}, \hat{y}) d t$, we have $g_{\lambda}(x, y)=\sum_{\gamma \in \Gamma} G_{\lambda}(\hat{x}, \gamma \hat{y})$. Hence $\lambda(\Gamma)$ is the supremum of those $\lambda$ for which $\sum_{\gamma \in \Gamma} G_{\lambda}(x, \gamma y)$ converges for all $x \neq y, x, y \in H$. Also $\delta(\Gamma)$ is the supremum of those $s$ for which the series $\sum_{\gamma \in \Gamma} e^{-s d(x, \gamma y)}$ diverges. In the general variable curvature case, since one does not have a simple qualitative connection between $G_{\lambda}(x, y)$ and $e^{-d(x, y)}$, one should not expect that $\delta(\Gamma)$ and $\lambda(\Gamma)$ can be related 
by any equality or inequality. This question is completely settled in the constant curvature case by Sullivan ([S3]) and in the symmetric space case by Corlette ([Co]). We want to point out that their results can be generalized to any $H$ which is harmonic with a compact quotient.

Theorem (6.6.1). Let $H$ be a simply connected harmonic manifold with pinched negative curvature and a compact quotient. Let $\Gamma$ be any Fuchsian group of $H$. Then we have:

(i)

$$
\lambda(\Gamma)=\left\{\begin{array}{ll}
\frac{1}{4} h^{2}, & \text { if } \delta(\Gamma) \leq \frac{h}{2} \\
\delta(\Gamma)(h-\delta(\Gamma)), & \text { if } \delta(\Gamma) \geq \frac{h}{2}
\end{array}, \quad \text { where } h=\lim _{R \rightarrow \infty} \frac{\log \operatorname{Vol} B(x, R)}{R} .\right.
$$

(ii) $M$ is $\lambda(\Gamma)$ recurrent (i.e. $\left.\int_{0}^{\infty} e^{t \lambda(\Gamma)} H_{t}(x, y) d t=\infty\right)$ if and only if $\delta(\Gamma) \geq \frac{h}{2}$ and the Poincaré series diverges at $s=\delta(\Gamma)$.

Proof. (i) If $H$ is harmonic with a compact quotient, then it is asymptotically harmonic ([Yu2]) and consequently, there are positive $\lambda$-harmonic functions which satisfy $\varphi(x, y)=\varphi(R), R=d(x, y)$ and moreover

$$
\ddot{\varphi}(R)-\frac{A^{\prime}(R)}{A(R)} \dot{\varphi}(R)+\lambda \varphi=0
$$

where $A(R)=$ the area of the geodesic sphere $S(x, R)$.

For $R$ near 0 and infinity respectively, this equation becomes

$$
\begin{aligned}
R=0: & \ddot{\varphi}(R)-\frac{n}{R} \dot{\varphi}(R)+\lambda \varphi=0, \quad n=\operatorname{dim} H . \\
R=\infty: \ddot{\varphi}(R)-h \dot{\varphi}(R)+\lambda \varphi=0, & \left.\quad \text { (by asymptotic harmonicity, } \lim _{R \rightarrow \infty} \frac{A^{\prime}(R)}{A(R)}=h\right) .
\end{aligned}
$$

By the same argument as in [S3], it is easy to see that the Green kernel $G_{\lambda}(x, y)$ satisfies

$$
G_{\lambda}(x, y) \sim e^{-\left(\frac{h}{2}+\sqrt{\frac{h^{2}}{4}-\lambda}\right) d(x, y)} .
$$

Thus $\lambda(\Gamma)$ is the supremum of all $\lambda$ such that the series

$$
\sum_{\gamma \in \Gamma} e^{-\left(\frac{h}{2}+\sqrt{\frac{h^{2}}{4}-\lambda}\right) d(x, y)}
$$

converges for $x, y \in H$. If $\delta(\Gamma) \leq \frac{h}{2}$, then the above series converges for all $0 \leq$ $\lambda<\frac{h^{2}}{4}$ and consequently $\lambda(\Gamma)=\frac{h^{2}}{4}$. If $\delta(\Gamma)>\frac{h}{2}$ then it is easy to see that the supremum value of $\lambda$ such that $\frac{h}{2}+\sqrt{\frac{h^{2}}{4}-\lambda} \geq \delta(\Gamma)$ is exactly $\lambda=\delta(\Gamma)(h-\delta(\Gamma))$. (ii) follows easily from (i).

Remark. Recall that in the construction of the $\lambda(\Gamma)$-harmonic density (see section (4.2)), one requires that $\lambda(\Gamma)<\frac{h^{2}}{4}$. If $H$ is a harmonic manifold then the harmonic density coincides with the conformal density and it provides no new information. Only for non-harmonic manifolds does the harmonic density bring new information about the bottom spectrum. 
We end this section by recalling extraordinary rigidity properties for the discrete isometry groups acting on quaternionic hyperbolic space $H_{\mathbb{H}}^{n}$ or the Cayley plane $H_{C_{a}}^{2}$.

Theorem (6.6.2) (Corlette $[\mathrm{Co}]$ ). (i) If $\Gamma$ is a Fuchsian group on $H_{\mathbb{H}}^{n}, n \geq 2$, then $\delta(\Gamma)=4 n+2$ or $\delta(\Gamma) \leq 4 n$. If $\delta(\Gamma)=4 n+2$, then $\Gamma$ is a lattice.

(ii) If $\Gamma$ is a Fuchsian group on $H_{C_{a}}^{2}$, then $\delta(\Gamma)=22$ or $\delta(\Gamma) \leq 16$. If $\delta(\Gamma)=22$ then $\Gamma$ is a lattice.

\section{Mostow Rigidity in A General SETting}

(7.1) Nonsingularity, cross ratio, and conformality. Suppose that $M_{1}=$ $H_{1} / \Gamma_{1}$ and $M_{2}=H_{2} / \Gamma_{2}$ are two Fuchsian manifolds. Assume that $\varphi: \Gamma_{1} \rightarrow \Gamma_{2}$ is a homomorphism and $\Phi: \Lambda_{1}=L\left(\Gamma_{1}\right) \rightarrow \Lambda_{2}=L\left(\Gamma_{2}\right)$ is a Borel map conjugating the action of $\Gamma_{1}$ to that of $\Gamma_{2}$ : $\Phi(\gamma \zeta)=\varphi(\gamma)(\Phi(\zeta))$ for all $\gamma \in \Gamma_{1}$ and $\zeta \in \Lambda_{1}$. For any Hadamard manifold $H$ we define, for $x \in H$ and $\zeta_{1}, \zeta_{2}, \zeta_{3}, \zeta_{4} \in \partial H$, the function

$$
r_{x}\left(\zeta_{1}, \zeta_{2}, \zeta_{3}, \zeta_{4}\right)=\frac{e^{-\beta_{x}\left(\zeta_{1}, \zeta_{2}\right)} e^{-\beta_{x}\left(\zeta_{3}, \zeta_{4}\right)}}{e^{-\beta_{x}\left(\zeta_{1}, \zeta_{3}\right)} e^{-\beta_{x}\left(\zeta_{2}, \zeta_{4}\right)}}
$$

where $\beta_{x}$ is the Busemann cocycle (see (1.2.2)). It is easy to see that $r_{x}$ does not depend on the choice of the base point $x$, so we denote it by $r$. We call $r\left(\zeta_{1}, \zeta_{2}, \zeta_{3}, \zeta_{4}\right)$ the cross ratio of $\zeta_{1}, \zeta_{2}, \zeta_{3}, \zeta_{4}$. This cross ratio coincides with the usual cross ratio in the constant curvature case.

Theorem (7.1.1). Suppose that $\delta\left(\Gamma_{1}\right)=\delta\left(\Gamma_{2}\right) \triangleq \delta$ and that $\Phi$ is nonsingular with respect to the $\delta$-conformal densities $\sigma^{1}$ of $\Gamma_{1}$ and $\sigma^{2}$ of $\Gamma_{2}$. Suppose also that the Poincaré series for $\Gamma_{1}$ diverges at $\delta$. Then $\Phi$ preserves the cross ratio almost everywhere.

Proof. The idea of the following proof is due to Sullivan ([S2]). The map $\Phi \times \Phi$ is a measurable conjugacy between the action $\Gamma_{1}$ on $\Lambda_{1} \times \Lambda_{1}$ and $\Gamma_{2}$ on $\Lambda_{2} \times \Lambda_{2}$. Let $U^{\sigma^{i}}$ be the $\Gamma_{i}$-invariant measure on $\Lambda_{i} \times \Lambda_{i}$ that we construct in (5.2). The $(\Phi \times \Phi)^{*}\left(U^{\sigma^{2}}\right)$ is a $\Gamma_{1}$-invariant measure on $\Lambda_{1} \times \Lambda_{1}$ absolutely continuous with respect to $U^{\sigma^{1}}$ (since $\Phi:\left(\Lambda_{1}, \sigma^{1}\right) \rightarrow\left(\Lambda_{2}, \sigma^{2}\right)$ is nonsingular). Because the Poincaré series for $\Gamma_{1}$ diverges at $\delta$, the $\Gamma_{1}$ action on $\Lambda_{1} \times \Lambda_{1}$ is ergodic with respect to $U^{\sigma^{1}}$. Thus the $\Gamma_{1}$-invariant function $f=\frac{d(\Phi \times \Phi)^{*}\left(U^{\sigma^{2}}\right)}{d U^{\sigma^{1}}}$ must be constant almost everywhere on $\Lambda_{1} \times \Lambda_{1}$. Therefore $\Gamma_{2}$ also acts ergodically with respect to $U^{\sigma^{2}}$. If we denote $h(\zeta)=\frac{d \Phi^{*}\left(\sigma_{y}^{2}\right)}{d \sigma_{x}^{1}}(\zeta)$, where $x \in H_{1}, y \in H_{2}$, then we have:

$$
\begin{aligned}
d(\Phi \times \Phi)^{*}\left(U^{\sigma^{2}}\right)(\zeta, \eta) & =e^{-\delta \beta_{y}(\varphi \zeta, \varphi \eta)} d \Phi^{*}\left(\sigma_{y}^{2}\right)(\zeta) d \Phi^{*}\left(\sigma_{y}^{2}\right)(\eta) \\
& =e^{-\delta \beta_{y}(\varphi \zeta, \varphi \eta)} h(\zeta) h(\eta) d \sigma_{x}^{1}(\zeta) d \sigma_{x}^{1}(\eta) .
\end{aligned}
$$

However $(\Phi \times \Phi)^{*}\left(U^{\sigma^{2}}\right)=c U^{\sigma^{1}}$ for some constant $c$. So we obtain

$$
e^{\delta \beta_{y}(\varphi \zeta, \varphi \eta)} h(\zeta) h(\eta)=c e^{\delta \beta_{x}(\zeta, \eta)},
$$

from which it follows easily that $\Phi$ preserves the cross ratio almost everywhere.

Remark. It is easy to see from the proof that, without the assumption $\delta\left(\Gamma_{1}\right)=$ $\delta\left(\Gamma_{2}\right)$, one can get

$$
r\left(\zeta_{1}, \zeta_{2}, \zeta_{3}, \zeta_{4}\right)=r\left(\Phi \zeta_{1}, \Phi \zeta_{2}, \Phi \zeta_{3}, \Phi \zeta_{4}\right)^{\frac{\delta\left(\Gamma_{2}\right)}{\delta\left(\Gamma_{1}\right)}} .
$$


However, one can always make a homothetic change of the Riemannian metric on $H_{2}$, such that under the new metric, $\delta\left(\Gamma_{2}\right)=\delta\left(\Gamma_{1}\right)$.

Theorem (7.1.2). If $\varphi: \Gamma_{1} \rightarrow \Gamma_{2}$ is a homomorphism between two Fuchsian groups and $\Phi: L\left(\Gamma_{1}\right) \rightarrow L\left(\Gamma_{2}\right)$ a Borel map conjugating the action of $\Gamma_{1}$ to that of $\Gamma_{2}$. Assume that the Poincaré series of $\Gamma_{1}$ diverges at $\delta\left(\Gamma_{1}\right)$ and $\delta\left(\Gamma_{2}\right)=\delta\left(\Gamma_{1}\right)$. Then $\Phi$ is conformal almost everywhere with respect to the Busemann metric.

Proof. By the continuity of the conformal measures and the fact that $\Phi$ preserves cross ratio almost everywhere with respect to the conformal measure, it is easy to see that $\Phi$ coincides almost everywhere with a continuous map $\tilde{\Phi}: L\left(\Gamma_{1}\right) \rightarrow L\left(\Gamma_{2}\right)$ which preserves cross ratio for all four-tuples. Now fix $x \in H_{1}, y \in H_{2}$ and $\zeta, \eta_{1} \in L\left(\Gamma_{1}\right)$. Let $-\zeta$ be the geodesic reflection of $\zeta$ with respect to $x$. Then we have, for all $\eta \in L\left(\Gamma_{1}\right)$,

$$
\frac{e^{-\beta_{y}(\tilde{\Phi} \zeta, \tilde{\Phi} \eta)}}{e^{-\beta_{x}(\zeta, \eta)}}=\frac{e^{-\beta_{x}\left(-\zeta, \eta_{1}\right)} e^{-\beta_{y}(\tilde{\Phi} \zeta, \tilde{\Phi}(-\zeta))} e^{-\beta_{y}\left(\tilde{\Phi} \eta, \tilde{\Phi} \eta_{1}\right)}}{e^{-\beta_{y}\left(\tilde{\Phi}(-\zeta), \tilde{\Phi} \eta_{1}\right)} e^{-\beta_{x}\left(\eta, \eta_{1}\right)}}
$$

Hence

$$
\lim _{\eta \rightarrow \zeta} \frac{e^{-\beta_{y}(\tilde{\Phi} \zeta, \tilde{\Phi} \eta)}}{e^{-\beta_{x}(\zeta, \eta)}}=\frac{e^{-\beta_{x}\left(-\zeta, \eta_{1}\right)} e^{-\beta_{y}(\tilde{\Phi} \zeta, \tilde{\Phi}(-\zeta))} e^{-\beta_{y}\left(\tilde{\Phi} \zeta, \tilde{\Phi} \eta_{1}\right)}}{e^{-\beta_{y}\left(\tilde{\Phi}(-\zeta), \tilde{\Phi} \eta_{1}\right)} e^{-\beta_{x}\left(\zeta, \eta_{1}\right)}} .
$$

Therefore $\tilde{\Phi}$ is conformal with respect to the Busemann metric.

\section{(7.2) Time-preserving conjugacy of the geodesic flows.}

Theorem (7.2.1). Let $\varphi$ be a homomorphism between two Fuchsian groups and $\Phi: L\left(\Gamma_{1}\right) \rightarrow L\left(\Gamma_{2}\right)$ be a Borel map conjugating the action of $\Gamma_{1}$ to that of $\Gamma_{2}$. Assume that the Poincaré series for $\Gamma_{1}$ diverges at $\delta\left(\Gamma_{1}\right)$. Assume also that $\delta\left(\Gamma_{1}\right)=$ $\delta\left(\Gamma_{2}\right) \triangleq \delta$ and that $\Phi$ is nonsingular with respect to the $\delta$-conformal densities. Then there exists a continuous map $\Omega\left(\Gamma_{1}\right) \stackrel{f}{\rightarrow} \Omega\left(\Gamma_{2}\right)$ between the nonwandering sets of the geodesic flows on $M_{1}$ and $M_{2}$ such that $f$ is a time-preserving conjugacy of the geodesic flows on $\Omega\left(\Gamma_{1}\right)$ and $\Omega\left(\Gamma_{2}\right)$.

Proof. i) We give the construction of the conjugacy following $[\mathrm{H}]$ where the cocompact case is treated. We denote by $\mu^{\sigma^{1}}$ and $\mu^{\sigma^{2}}$ the Bowen-Margulis measure associated to $\sigma^{1}$ and $\sigma^{2}$, normalized such that $\mu^{\sigma^{1}}\left(\Omega\left(\Gamma_{1}\right)\right)=\mu^{\sigma^{2}}\left(\Omega\left(\Gamma_{2}\right)\right)=1$. Let $\mu_{1}^{s u}$ (resp. $\mu_{2}^{s u}$ ) be the Margulis conditional measures as constructed in section (5.2). Then they can also be viewed as measures at infinity via the canonical projection $P: W^{s u}(v) \rightarrow \partial H \backslash\{v(-\infty)\}: w \rightarrow w(\infty)$. Now we lift everything to the universal cover. For each $v \in \Omega\left(\Gamma_{1}\right)$, pick up $w \in \Omega\left(\Gamma_{2}\right)$ such that $\Phi(v(-\infty), v(\infty))=$ $(w(-\infty), w(\infty))$. Then there exists a Borel function $f_{v, w}$ on $\lambda\left(\Gamma_{2}\right)$ such that $\Phi\left(\mu_{v}^{s u}\right)=f_{v, w} \mu_{w}^{s u}$. Since $\mu_{g^{t} v}^{s u}=e^{\delta t} \mu_{v}^{s u}, \mu_{g^{t} w}^{s u}=e^{\delta t} \mu_{w}^{s u}$, there is for every $v \in \Omega\left(\Gamma_{1}\right)$ a unique $f(v) \in \Omega\left(\Gamma_{2}\right)$ such that $\Phi(v(-\infty), v(\infty))=(f(v)(-\infty), f(v)(+\infty))$ and that $f_{v, w}(f(v)(\infty))=1$. Clearly $f\left(g^{t} v\right)=g^{t}(f(v))$ and $f$ commutes with the action of $\Gamma$. It is also clear that $f$ preserves the $W^{u}$ foliation. Moreover since the measure $d \mu^{u}=d \mu^{s u} \times d t$ is holonomy invariant under the $W^{s s}$ foliation, $f$ also preserves the $W^{s s}$ foliation. Thus $f$ descends to a time-preserving conjugacy of the geodesic flows on $\Omega\left(\Gamma_{1}\right)$ and $\Omega\left(\Gamma_{2}\right)$. If we choose $\Phi$ to be continuous at the beginning (see the proof of Theorem (7.1.1) and the discussion there), then $f$ is also continuous. 
Theorem (7.2.2). Suppose that $\Gamma_{1}$ and $\Gamma_{2}$ are convex cocompact groups and that $\varphi: \Gamma_{1} \rightarrow \Gamma_{2}$ is an isomorphism. Then there is a unique homeomorphism $\Phi$ : $L\left(\Gamma_{1}\right) \rightarrow L\left(\Gamma_{2}\right)$ which conjugates the action of $\Gamma_{1}$ to that of $\Gamma_{2}$. Moreover, the following properties are equivalent:

i) $\delta\left(\Gamma_{1}\right)=\delta\left(\Gamma_{2}\right) \triangleq \delta$, $\Phi$ is nonsingular with respect to the $\delta$-conformal densities $\sigma^{1}$ and $\sigma^{2}$.

ii) $\Phi$ preserves the cross ratio.

iii) $\delta\left(\Gamma_{1}\right)=\delta\left(\Gamma_{2}\right) \triangleq \delta$ and $\Phi$ is conformal with respect to the Busemann metric.

iv) $M_{1}=H_{1} / \Gamma_{1}$ and $M_{2}=H_{2} / \Gamma_{2}$ have the same marked length spectrum.

v) There exists a time-preserving conjugacy of the geodesic flows on $\Omega\left(\Gamma_{1}\right)$ and $\Omega\left(\Gamma_{2}\right)$.

Proof. Since convex cocompact groups are hyperbolic groups in the sense of Gromov, and any isomorphism between two hyperbolic groups is a quasi-isometry with respect to their word metrics, hence the isomorphism must induce a homeomorphism between their limit sets. Now i) $\Rightarrow$ ii) is Theorem (7.1.1). ii) $\Rightarrow$ iii) is Theorem (7.1.2). iii) $\Rightarrow \mathrm{i}$ ): This is because, in the convex cocompact case, the conformal density $\sigma^{1}$ (resp. $\sigma^{2}$ ) is exactly the $\delta$-dimensional Hausdorff measure with respect to the Busemann metric. Thus the conformal map $\Phi$ preserves the measure class of the conformal density. i) $\Rightarrow \mathrm{v}$ ) is Theorem (7.2.1). v) $\Rightarrow$ iv) is obvious. iv) $\Rightarrow \mathrm{i})$ : The proof is the same as the cocompact case, which can be found in $[\mathrm{H}]$.

\section{ACKNOWLEDGMENTS}

I would like to thank Anatoly Katok for his constant encouragement and interest. This work was started when I was enjoying the hospitality of SFB 170 at Göttingen, where I had beneficial conversations with Professor G. Knieper. I am grateful to MSRI where most part of this work was completed. I would also like to thank the referee for a couple of helpful suggestions.

\section{REFERENCES}

[Ah] L. V. Ahlfors, Fundamental polyhedrons and limit point sets of Kleinian Groups, Proc. Nat. Acad. Sci. U.S.A. 55 (1966), 251-254. MR 32:5844

[A1] A. Ancona, Sur les fonctions propres positives des variétés de Cartan-Hadamard, Comm. Math. Helv. 64 (1989), 62-83. MR 90k:53069

[A2] A. Ancona, Théorie du potentiel sur les graphes et les variétés, Lect. Notes in Math. 1427, Springer, Berlin, 1990. MR 92g:31012

[A3] A. Ancona, Negatively curved Manifolds, Elliptic operators, and the Martin boundary, Ann. Math. 121 (1987), 495-536. MR 88k:53069

[And] M. Anderson, The Dirichlet problem at infinity, J. Diff. Geom. 18 (1983), 701-721. MR 85m:58178

[AS] M. Anderson and R. Schoen, Positive harmonic functions on complete manifolds of negative curvature, Ann. Math. 121 (1985), 429-461. MR 87a:58151

[BGS] W. Ballmann, M. Gromov and V. Schroeder, Manifolds of non-positive curvature, Progress in Mathematics 61, Birkhäuser (1985). MR 87h:53050

[B] B. H. Bowditch, Geometrical finiteness with variable negative curvature, I.H.E.S. Preprint.

[C] M. Coornaert, Sur les groups proprement discontinus d'isometriés des espaces hyperboliques au sens de Gromov, Thèse (1990).

[Co] K. Corlette, Hausdorff dimension of limit sets I, Invent. Math. 102 (1990), 521-542. MR 91k:58067

[E1] P. Eberlein, Geodesic flows on negatively curved manifolds; I, Ann. of Math. 95 (1972), 151-170. MR 46:10024 
[E2] P. Eberlein, Geodesic flows on negatively curved manifolds; II, Trans. of AMS 178 (1973), 57 - 82. MR 47:2636

[E-O'N] P. Eberlein and B. O'Neil, Visibility manifolds, Pacific J. Math 46 (1973), 45-109. MR 49:1241

[GM] W.M. Goldman and J.J. Millson, Local rigidity of discrete groups acting on complex hyperbolic spaces, Invent. Math. 88 (1987), 495-520. MR 88f:22027

[G] M. Gromov, Hyperbolic groups, Essays in group theory, Springer, 1987. MR 89e:20070

$[\mathrm{H}] \quad$ U. Hamenstädt, Time preserving conjugacies of geodesic flows, Erg. Th. and Dyn. Sys. 12 (1992), 67-74. MR 93g:58115

[K] V. A. Kaimanovich, Invariant measures for the geodesic flow and measures at infinity on negatively curved manifolds, Ann. Inst. Henri Poincaré, Physique Théorique 53, no. 4 (1990), 361-393. MR 92b:58176

[KL] Y. Kifer and F. Ledrappier, Hausdorff dimension of harmonic measures on negatively curved manifolds, Trans. of AMS 318, No. 2 (1990), 685 - 704. MR 91a:58205

[L1] F. Ledrappier, Ergodic properties of Brownian motion on covers of compact negatively curved manifolds, Bol. Soc. Bras. Mat. 19 (1988), 115-140. MR 91e:58210

[L2] F. Ledrappier, Harmonic measures and Bowen-Margulis measures, Israel J. Math. 71 (1990), 275-287. MR 92a:58107

[LY] F. Ledrappier and L. S. Young, The metric entropy of diffeomorphisms I, Ann. of Math. 122 (1985), 509-539. MR 87i:58101a

[M] G. A. Margulis, Thesis, In Russian, Moscow University, 1970.

[M1] B. Marcus, Unique ergodicity of the horocycle flow: variable negative curvature case, Israel J. Math. 21 (1975), 133-144. MR 54:1302

[N] P. J. Nicholls, The ergodic theory of discrete groups, Cambridge University Press, 1989. MR 91i: 58104

[Pan] P. Pansu, Quasiisométriés des variétés à courbure négative, Thèse Université de Paris 7 (1987).

[P] S. J. Patterson, Measures on limit sets of Kleinian groups, Analytical and geometric aspects of hyperbolic space, Cambridge University Press, 1987, pp. 291-323. MR 89b:58122

[R] M. Ratner, Raghunathan's conjectures for $S L(2, R)$, Israel J. Math. 80 (1992), 1-37. MR 94k:22024

[Si1] Y. G. Sinai, Classical systems with Lebesgue spectrum II, AMS. Transl. 268 (1968), 34-88.

[Si2] Y. G. Sinai, Gibbs measure in ergodic theory, Russ. Math. Surveys 27, no. 4 (1972), 21-69. MR 53:3265

[S1] D. Sullivan, The density at infinity of a discrete group of hyperbolic motions, Publ. Math. I.H.E.S. 50 (1979), 171-202. MR 81b:58031

[S2] D. Sullivan, Discrete conformal groups and measurable dynamics, Bull. Amer. Math. Soc. 6 (1982), 57-73. MR 83c:58066

[S3] D. Sullivan, Related aspects of positivity in Riemannian geometry, J. Diff. Geom. 25 (1987), 327-351. MR 88d:58132

[S4] D. Sullivan, Disjoint spheres, diophantine approximation, and the logarithm law for geodesics, Acta Math. 149 (1982), 215-237. MR 84j:58097

[Y] L. S. Young, Dimension, entropy and Lyapunov exponents, Erg. Th. Dynam. Sys. 2 (1982), 109-129. MR 84h:58087

[Yu1] C. B. Yue, Brownian motion on Ansov foliations and manifolds of negative curvature, J. of Diff. Geom. 41 (1995), 159-183. MR 95k:58123

[Yu2] C. B. Yue, On Sullivan's conjecture, Random and Comp. Dyn. 1 (1992), 131-145.

[Yu3] C. B. Yue, Dimension and rigidity of quasi-Fuchsian representations, Annals of Mathematics 143 (1996), 331-355. CMP 96:10

[Yu4] C. B. Yue, Mostow rigidity for rank 1 discrete subgroups with ergodic Bowen-Margulis measure, To appear in Inv. Math.

Department of Mathematics, Pennsylvania State University, University Park, PennSYLVANIA 16802

E-mail address: yue@math.psu.edu 